\title{
AGT, Burge pairs and minimal models
}

\section{Bershtein ${ }^{a, b, c}$ and O. Foda ${ }^{d}$}

${ }^{a}$ Landau Institute for Theoretical Physics, Chernogolovka, Russia

${ }^{b}$ Institute for Information Transmission Problems, Moscow, Russia

${ }^{c}$ National Research University Higher School of Economics, International Laboratory of Representation Theory and Mathematical Physics, Independent University of Moscow, Moscow, Russia

${ }^{d}$ Mathematics and Statistics, University of Melbourne, Parkville, VIC 3010, Australia

E-mail: mbersht@gmail.com, omar.foda@unimelb.edu.au

Abstract: We consider the AGT correspondence in the context of the conformal field theory $\mathcal{M}^{p, p^{\prime}} \otimes \mathcal{M}^{\mathcal{H}}$, where $\mathcal{M}^{p, p^{\prime}}$ is the minimal model based on the Virasoro algebra $\mathcal{V}^{p, p^{\prime}}$ labeled by two co-prime integers $\left\{p, p^{\prime}\right\}, 1<p<p^{\prime}$, and $\mathcal{M}^{\mathcal{H}}$ is the free boson theory based on the Heisenberg algebra $\mathcal{H}$. Using Nekrasov's instanton partition functions without modification to compute conformal blocks in $\mathcal{M}^{p, p^{\prime}} \otimes \mathcal{M}^{\mathcal{H}}$ leads to ill-defined or incorrect expressions.

Let $\mathcal{B}_{n}^{p, p^{\prime}, \mathcal{H}}$ be a conformal block in $\mathcal{M}^{p, p^{\prime}} \otimes \mathcal{M}^{\mathcal{H}}$, with $n$ consecutive channels $\chi_{\iota}$, $\iota=1, \cdots, n$, and let $\chi_{\iota}$ carry states from $\mathcal{H}_{r_{\iota}, s_{\iota}}^{p, p^{\prime}} \otimes \mathcal{F}$, where $\mathcal{H}_{r_{\iota}, s_{\iota}}^{p, p^{\prime}}$ is an irreducible highestweight $\mathcal{V}^{p, p^{\prime}}$-representation, labeled by two integers $\left\{r_{\iota}, s_{\iota}\right\}, 0<r_{\iota}<p, 0<s_{\iota}<p^{\prime}$, and $\mathcal{F}$ is the Fock space of $\mathcal{H}$.

We show that restricting the states that flow in $\chi_{\iota}, \iota=1, \cdots, n$, to states labeled by partition pairs $\left\{Y_{1}^{\iota}, Y_{2}^{\iota}\right\}$ that satisfy $Y_{2, \sigma}^{\iota, \top}-Y_{1, \sigma+r_{\iota}-1}^{\iota, T} \geqslant 1-s_{\iota}$, and $Y_{1, \sigma}^{\iota, T}-Y_{2, \sigma+p-r_{\iota}-1}^{\iota, T} \geqslant$ $1-p^{\prime}+s_{\iota}$, where $Y_{i, \sigma}^{\iota, \mathrm{T}}$ is the $\sigma$-column of $Y_{i}^{\iota}, i \in\{1,2\}$, we obtain a well-defined expression that we identify with $\mathcal{B}_{n}^{p, p^{\prime}, \mathcal{H}}$. We check the correctness of this expression for 1. Any 1point $\mathcal{B}_{1}^{p, p^{\prime}, \mathcal{H}}$ on the torus, when the operator insertion is the identity, and 2 . The 6-point $\mathcal{B}_{3}^{3,4, \mathcal{H}}$ on the sphere that involves six Ising magnetic operators.

KEYwords: Supersymmetric gauge theory, Conformal and W Symmetry

ARXIV EPRINT: 1404.7075 


\section{Contents}

1 Introduction $\quad 1$

1.1 AGT in generic models 1

1.2 AGT in minimal models 1

1.3 Zeros in denominators and deformations 2

1.4 Zeros in denominators and restrictions 2

1.5 Unrestricted partition pairs 2

1.6 Restricted partition pairs 3

1.7 Outline of contents 3

2 The instanton partition function $\quad 4$

2.1 Partitions 4

2.2 Partition pairs 4

2.3 A decomposition of the instanton partition function 5

2.4 The building block of the instanton partition function 5

2.4.1 The 2-component vector $\vec{a}^{\iota}=\left\{a^{\iota},-a^{\iota}\right\} \quad 6$

2.4.2 The partition pairs $\vec{Y}$ and $\vec{W} \quad 6$

$\begin{array}{lll}2.4 .3 & \text { The scalar } \mu^{\iota} & 6\end{array}$

2.4.4 The denominator 6

$\begin{array}{ll}2.4 .5 & \text { The numerator }\end{array}$

$\begin{array}{lll}2.4 .6 & \text { Remark } & 7\end{array}$

$\begin{array}{lll}2.4 .7 & \text { Normalisation } & 7\end{array}$

3 Unrestricted instanton partition functions for $\mathcal{M}^{p, p^{\prime}, \mathcal{H}} \quad 7$

$\begin{array}{lll}3.1 & \text { AGT parameterisation. Generic models } & 7\end{array}$

3.2 AGT parameterisation. Minimal models 8

3.3 Charge content 8

3.4 Unrestricted instanton partition functions give incorrect 1-point functions $\begin{array}{ll}\text { on the torus } & 8\end{array}$

4 Restricted instanton partition functions for $\mathcal{M}^{p, p^{\prime}, \mathcal{H}}$. The denominator 9

$\begin{array}{ll}4.1 \text { More notation } & 10\end{array}$

$\begin{array}{lll}4.2 & \text { Two zero-conditions } & 10\end{array}$

$\begin{array}{lll}4.3 & \text { From two zero-conditions to one zero-condition } & 10\end{array}$

$\begin{array}{ll}4.4 \text { One non-zero condition } & 11\end{array}$

$\begin{array}{lll}\text { 4.4.1 Remark } & 11\end{array}$

4.5 Products that appear in the denominator 11

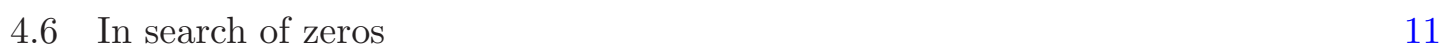

$4.7\left\{Y_{1}, Y_{1}\right\}_{\text {den }} \quad 11$

$4.8\left\{Y_{1}, Y_{1}\right\}^{\prime},\left\{Y_{2}, Y_{2}\right\}_{\text {den }}$ and $\left\{Y_{2}, Y_{2}\right\}_{\text {den }}^{\prime} \quad 11$

$4.9\left\{Y_{1}, Y_{2}\right\}_{\text {den }} \quad 11$ 
4.9.1 From two zero-conditions to one non-zero-condition 12

$\begin{array}{ll}\text { 4.9.2 The stronger condition } & 12\end{array}$

$4.10\left\{Y_{2}, Y_{1}\right\}_{\text {den }} \quad 12$

4.10.1 From two zero-conditions to one non-zero-condition 12

$\begin{array}{ll}4.10 .2 \text { The stronger condition } & 12\end{array}$

$4.11\left\{Y_{1}, Y_{2}\right\}_{\text {den }}^{\prime} \quad 13$

$4.12\left\{Y_{2}, Y_{1}\right\}_{\text {den }}^{\prime} \quad 13$

4.13 Restricted instanton partition functions give the correct 1-point function on $\begin{array}{ll}\text { the torus } & 13\end{array}$

$\begin{array}{lll}4.13 .1 \text { Remark } & 14\end{array}$

5 Restricted instanton partition functions for $\mathcal{M}^{p, p^{\prime}, \mathcal{H}}$. The numerator 14

$\begin{array}{lll}5.1 \text { Products that appear in the numerator } & 14\end{array}$

$\begin{array}{ll}5.2 \text { Notation } & 14\end{array}$

$\begin{array}{lll}5.3 & \text { The fusion rules } & 14\end{array}$

5.4 Bounds on $M_{[ \pm, \pm, \pm]}$and $N_{[ \pm, \pm, \pm]} \quad 15$

$\begin{array}{ll}5.5\left\{Y_{1}, W_{1}\right\}_{\text {num }} & 16\end{array}$

$\begin{array}{lll}5.6 & \left\{Y_{1}, W_{1}\right\}_{\text {num }}^{\prime} & 17\end{array}$

$\begin{array}{lll}\text { 5.7 The remaining six products } & 17\end{array}$

$\begin{array}{lll}5.7 .1 & \left\{Y_{2}, W_{2}\right\}_{\text {num }} & 17\end{array}$

$\begin{array}{lll}5.7 .2\left\{Y_{2}, W_{2}\right\}_{\text {num }}^{\prime} & 17\end{array}$

$\begin{array}{lll}5.7 .3\left\{Y_{1}, W_{2}\right\}_{\text {num }} & 17\end{array}$

$\begin{array}{lll}5.7 .4 & \left\{Y_{1}, W_{2}\right\}_{\text {num }}^{\prime} & 17\end{array}$

$\begin{array}{lll}5.7 .5 & \left\{Y_{2}, W_{1}\right\}_{\text {num }} & 17\end{array}$

$\begin{array}{lll}5.7 .6 & \left\{Y_{2}, W_{1}\right\}_{\text {num }}^{\prime} & 17\end{array}$

$\begin{array}{lll}5.7 .7 & \text { Remark } & 17\end{array}$

5.8 If the denominator is zero, then the numerator is zero 17

$\begin{array}{ll}\text { 5.8.1 }\left\{Y_{1}, W_{1}\right\}_{\text {num }} \text { and }\left\{Y_{2}, W_{1}\right\}_{\text {num }}^{\prime} & 18\end{array}$

$\begin{array}{ll}\text { 5.8.2 }\left\{Y_{1}, W_{2}\right\}_{\text {num }} \text { and }\left\{Y_{2}, W_{2}\right\}_{\text {num }}^{\prime} & 18\end{array}$

$\begin{array}{lll}5.8 .3\left\{Y_{2}, W_{1}\right\}_{\text {num }} \text { and }\left\{Y_{1}, W_{1}\right\}_{\text {num }}^{\prime} & 18\end{array}$

$\begin{array}{ll}\text { 5.8.4 }\left\{Y_{2}, W_{2}\right\}_{\text {num }} \text { and }\left\{Y_{1}, W_{2}\right\}_{\text {num }}^{\prime} & 18\end{array}$

$\begin{array}{ll}5.8 .5\left\{Y_{1}, W_{1}\right\}_{\text {num }}^{\prime} \text { and }\left\{Y_{1}, W_{2}\right\}_{\text {num }} & 18\end{array}$

$\begin{array}{ll}\text { 5.8.6 }\left\{Y_{2}, W_{1}\right\}_{\text {num }}^{\prime} \text { and }\left\{Y_{2}, W_{2}\right\}_{\text {num }} & 18\end{array}$

$\begin{array}{ll}5.8 .7\left\{Y_{1}, W_{2}\right\}_{\text {num }}^{\prime} \text { and }\left\{Y_{1}, W_{1}\right\}_{\text {num }} & 19\end{array}$

$\begin{array}{ll}5.8 .8\left\{Y_{2}, W_{2}\right\}_{\text {num }}^{\prime} \text { and }\left\{Y_{2}, W_{1}\right\}_{\text {num }} & 19\end{array}$

$\begin{array}{lll}6 & \text { An Ising conformal block } & 19\end{array}$

7 An explanation, based on a conjecture, of why we obtain $\mathcal{M}^{p, p^{\prime}, \mathcal{H}}$ conformal blocks

8 Generic model conformal blocks with Degenerate intermediate representations 
$\begin{array}{lll}9.1 & q-\mathfrak{g l}_{\infty} \text { Ding-Iohara } & 23\end{array}$

9.2 Higher-rank AGT-W 23

$\begin{array}{ll}9.3 & \text { The work of Alkalaev and Belavin } \\ 9.4\end{array}$

9.4 Previous works on AGT in minimal models 24

9.5 Geometry 24

\section{Introduction}

\subsection{AGT in generic models}

Consider the two-dimensional conformal field theory $\mathcal{M}^{\text {gen, } \mathcal{H}}=\mathcal{M}^{\text {gen }} \otimes \mathcal{M}^{\mathcal{H}}$, based on the algebra $\mathcal{V}^{\text {gen, } \mathcal{H}}=\mathcal{V}^{\text {gen }} \oplus \mathcal{H}$, where $\mathcal{M}^{\text {gen }}$ is a generic model with a chiral spectrum that spans infinitely-many infinite-dimensional irreducible highest-weight $\mathcal{V}^{\text {gen }}$-representations, ${ }^{1}$ $\mathcal{M}^{\mathcal{H}}$ is the conformal field theory of a free boson that takes values in $\mathbb{R}, \mathcal{V}^{\text {gen }}$ is the Virasoro algebra of generic central charge $c_{\text {gen }},{ }^{2}$ and $\mathcal{H}$ is the Heisenberg algebra. The Virasoro central charge of $\mathcal{M}^{\mathcal{H}}$ is $c_{\mathcal{H}}=1$. The AGT correspondence of Alday, Gaiotto and Tachikawa [1] identifies conformal blocks in $\mathcal{M}^{\text {gen, } \mathcal{H}}[2]$ with instanton partition functions in four-dimensional $\mathcal{N}=2$ supersymmetric quiver gauge theories [3]. Conjectured in [1], AGT was proven for $c_{\text {gen }}=1$ in [4], and for all $c_{\text {gen }}$ in [5] for conformal blocks with non-degenerate external primary fields.

\subsection{AGT in minimal models}

In this note, we consider AGT in the context of $\mathcal{M}^{p, p^{\prime}, \mathcal{H}}=\mathcal{M}^{p, p^{\prime}} \otimes \mathcal{M}^{\mathcal{H}}$, based on the algebra $\mathcal{V}^{p, p^{\prime}, \mathcal{H}}=\mathcal{V}^{p, p^{\prime}} \oplus \mathcal{H}$, where $\mathcal{M}^{p, p^{\prime}}$ is the minimal conformal field theory with a chiral spectrum that spans finitely-many $\mathcal{V}^{p, p^{\prime}}$ irreps, and $\mathcal{V}^{p, p^{\prime}}$ is the Virasoro algebra labeled by two co-prime integers $\left\{p, p^{\prime}\right\}, 0<p<p^{\prime}$, of central charge $c_{p, p^{\prime}}$,

$$
c_{p, p^{\prime}}=1-6\left(\left(\frac{p}{p^{\prime}}\right)^{\frac{1}{2}}-\left(\frac{p^{\prime}}{p}\right)^{\frac{1}{2}}\right)^{2}
$$

Let $\mathcal{B}_{n}^{p, p^{\prime}, \mathcal{H}}, \mathcal{B}_{n}^{p, p^{\prime}}$ and $\mathcal{B}^{\mathcal{H}}$ be conformal blocks with $n$ consecutive channels. ${ }^{3}$ We wish to compute any $\mathcal{B}_{n}^{p, p^{\prime}, \mathcal{H}}$ of vertex operators $\mathcal{O}_{\iota}^{p, p^{\prime}, \mathcal{H}}\left(z_{\iota}\right)=\mathcal{O}_{\iota}^{p, p^{\prime}}\left(z_{\iota}\right) \times \mathcal{O}_{\iota}^{\mathcal{H}}\left(z_{\iota}\right), \iota=0, \cdots, n+2$. Since any $\mathcal{B}_{n}^{p, p^{\prime}, \mathcal{H}}$ factorizes to $\mathcal{B}_{n}^{p, p^{\prime}} \times \mathcal{B}_{n}^{\mathcal{H}}$ and an explicit expression for the $\mathcal{M}^{\mathcal{H}}$-factor $\mathcal{B}_{n}^{\mathcal{H}}$ is known, ${ }^{4}$ computing $\mathcal{B}_{n}^{p, p^{\prime}, \mathcal{H}}$ is equivalent to computing its $\mathcal{M}^{p, p^{\prime}}$-factor $\mathcal{B}_{n}^{p, p^{\prime}}$ which is typically what we want.

\footnotetext{
${ }^{1}$ Only the chiral sector of a conformal field theory is discussed in this work, and this is implied in the sequel. We abbreviate 'the AGT correspondence' to 'AGT', and 'irreducible highest-weight representation' to 'irrep', which in this work is always infinite-dimensional.

${ }^{2}$ By generic Virasoro central charge $c_{\text {gen }}$ we specifically mean $c_{\text {gen }} \neq c_{p, p^{\prime}}$, where $c_{p, p^{\prime}}$ is the Virasoro central charge of the minimal model $\mathcal{M}^{p, p^{\prime}}$.

${ }^{3}$ Only linear conformal blocks, as in figure 3, are considered in this work. Our notation is such that an $n$-channel conformal block $\mathcal{B}_{n}^{\text {indices }}$, is the expectation value of $(n+3)$ vertex operators $\mathcal{O}_{\iota}^{\text {same indices }}\left(z_{\iota}\right)$, $\iota=0, \cdots,(n+2)$, in $\mathcal{M}^{\text {same indices }}$ on a Riemann surface $\mathcal{S}$, and $z_{\iota} \in \mathcal{S}$.

${ }^{4}$ See, for example, equation (1.9) in [5].
} 


\subsection{Zeros in denominators and deformations}

Applying AGT to minimal model conformal blocks without modification leads to ill-defined expressions, as will be explained in detail below. In particular, setting the parameters that appear in Nekrasov's partition functions to minimal model values leads to zeros in the denominators of the summands. Following [6], one can make the summands welldefined using suitable deformations of the parameters. Doing that, one finds whenever a denominator is zero in the limit of removing the deformations, the corresponding numerator is also zero in such a way that and that limit is well-defined. This is in agreement with [7], where arguments were given to the effect that, analytically continuing the conformal blocks in the conformal dimensions of the primary states that flow in each channel, the only singularities are poles and the sum of all residues is zero. This is the approach that was followed, albeit without discussion, in an earlier work on AGT in minimal models [8].

\subsection{Zeros in denominators and restrictions}

In this note, we follow a different approach from that discussed in subsection 1.3. Our idea is that the zeros in the denominators of Nekrasov's partition functions are due to including null states that should not be included. We avoid this by restricting the summations over Young diagrams that appear in Nekrasov partition functions to avoid these null states. We make the summands well-defined by restricting the partition pairs that label the summedover states to exclude the summands with poles. To compute $\mathcal{B}_{n}^{p, p^{\prime}, \mathcal{H}}$, the summations that label the factors in Nekrasov's instanton partition functions must be restricted to avoid ill-defined or incorrect expressions for $\mathcal{B}_{n}^{p, p^{\prime}, \mathcal{H}}$, and consequently for its $\mathcal{M}^{p, p^{\prime}}$-factor $\mathcal{B}_{n}^{p, p^{\prime}}$. Our approach allows us to characterise the Young diagrams that label the summands that do contribute to $\mathcal{B}_{n}^{p, p^{\prime}, \mathcal{H}}$.

\subsection{Unrestricted partition pairs}

The AGT expression for a linear conformal block $\mathcal{B}_{n}^{\text {gen }, \mathcal{H}}$, that has $n$ consecutive channels $\chi_{\iota}, \iota=1, \cdots, n$, is an $n$-fold sum,,$^{5}$

$$
\mathcal{B}_{n}^{\text {gen }, \mathcal{H}}=\sum_{\vec{Y}^{1}, \cdots, \vec{Y}^{n}} \prod_{\iota=1}^{n+1} q_{\iota}^{\left|\vec{Y}^{\iota}\right|} Z_{b b}^{\iota}\left(\vec{a}^{\iota-1}, \vec{Y}^{\iota-1}\left|\mu^{\iota}\right| \vec{a}^{\iota}, \vec{Y}^{\iota}\right),
$$

where the summand is a product of $(n+1)$ factors $q_{\iota}^{\left|\vec{Y}^{\iota}\right|} Z_{b b}^{\iota}\left[\vec{a}^{\iota-1}, \vec{Y}^{\iota-1}\left|\mu^{\iota}\right| \vec{a}^{\iota}, \vec{Y}^{\iota}\right], \iota=$ $1, \cdots, n+1$, that will be defined in section 2. Each factor $Z_{b b}^{\iota}$ is a rational function that depends on two pairs of 'unrestricted' Young diagrams $\left\{Y_{1}^{\iota-1}, Y_{2}^{\iota-1}\right\}$ and $\left\{Y_{1}^{\iota}, Y_{2}^{\iota}\right\}$. In other words, there are no conditions on these Young diagrams and all possible pairs are allowed. The denominator $z_{\mathrm{den}}^{\iota}$ of $Z_{b b}^{\iota}$ is a product of the norms of the states that flow in the preceding channel $\chi^{\iota-1}$ and the subsequent channel $\chi^{\iota}$. Since $Z_{b b}^{\iota}$ is labeled by unrestricted partition pairs, and the sums are over all possible unrestricted pairs, the states that flow in each channel belong to a Verma module of $\mathcal{V}^{\text {gen }, \mathcal{H}}$.

\footnotetext{
${ }^{5}$ The partition pairs $\vec{Y}^{0}$ and $\vec{Y}^{n+1}$ are trivial, that is they consist of empty partitions, and no summation is performed on them.
} 
Applying AGT without modification to $\mathcal{M}^{p, p^{\prime}, \mathcal{H}}$, one includes zero-norm states in the summation, and thereby includes states in a Verma module rather than in an irrep of $\mathcal{V}^{p, p^{\prime}, \mathcal{H}}$. This leads to summands in the instanton partition function with zero denominators. Further, as show, whenever a denominator in a summand vanishes, the corresponding numerator vanishes as well and one ends with ill-defined expressions

\subsection{Restricted partition pairs}

In this note, we consider $\mathcal{B}_{n}^{p, p^{\prime}, \mathcal{H}}$ as an instanton partition $Z_{\mathrm{Nek}}$ that consists of building block partition functions $Z_{b b}^{\iota}$ that has a numerator $z_{\text {num }}^{\iota}$ and a denominator $z_{\text {den }}^{\iota}, \iota=$ $1, \cdots, n+1$. $Z_{b b}^{\iota}$ connects two channels $\chi_{\iota-1}$ and $\chi_{\iota}$. The denominator $z^{\iota}$ of $Z_{b b}^{\iota}$ is a product of two factors $\left[z_{\text {norm }}^{\iota-1}\right]^{1 / 2}$ and $\left[z_{\text {norm }}^{\iota}\right]^{1 / 2}$ that account for the norms of the states that flow in the channels $\chi_{\iota-1}$ and $\chi \iota$, respectively. We characterise the zeros in these denominators that lead to ill-defined expressions for $\mathcal{B}_{n}^{p, p^{\prime}, \mathcal{H}}$. If channel $\chi_{\iota}, \iota=1, \cdots, n$, carries states that belong to an irreducible highest weight Virasoro representation that flows is $\mathcal{H}_{r_{\iota}, s_{\iota}}^{p, p^{\prime}}$, we attribute these zeros to the flow of null states that do not belong to $\mathcal{H}_{r_{\iota}, s_{\iota}}^{p, p^{\prime}}$, and eliminate these zeros by restricting the partition pairs that appear in Nekrasov's original expressions to partition pairs $\left\{Y_{1}, Y_{2}\right\}$, that satisfy the conditions

$$
Y_{2, \sigma}^{\iota, \top}-Y_{1, \sigma+r_{\iota}-1}^{\iota, \top} \geqslant 1-s_{\iota}, \quad Y_{1, \sigma}^{\iota, \top}-Y_{2, \sigma+p-r_{\iota}-1}^{\iota, \top} \geqslant 1-p^{\prime}+s_{\iota}
$$

where $Y_{i, \sigma}^{\iota, \top}$ is the $\sigma$-column of $Y_{i}^{\iota}, i \in\{1,2\}$. These restricted Burge pairs were first studied in [9] and appeared more recently in $[10,11]$. We show that when used to restrict AGT to compute $\mathcal{B}_{n}^{p, p^{\prime}, \mathcal{H}}$, that is when we sum over Burge pairs rather than on all possible partition pairs,

$$
\mathcal{B}_{n}^{p, p^{\prime}, \mathcal{H}}=\sum_{\vec{Y}^{1}, \cdots, \vec{Y}^{n}}^{\prime} \prod_{\iota=1}^{n+1} q_{\iota}^{\left|\vec{Y}^{\iota}\right|} Z_{b b}^{\iota}\left(\vec{a}^{\iota-1}, \vec{Y}^{\iota-1}\left|\mu^{\iota}\right| \vec{a}^{\iota}, \vec{Y}^{\iota}\right)
$$

where $\sum^{\prime}$ indicates that the sum is restricted to partition pairs that satisfy the Burge conditions (1.3), we obtain well-defined expressions. We check these expressions in two cases 1 . Any 1-point $\mathcal{B}_{1}^{p, p^{\prime}, \mathcal{H}}$ on the torus, when the operator insertion is the identity, and 2. The 6-point $\mathcal{B}_{3}^{3,4, \mathcal{H}}$, when all operator insertions involve Ising magnetic operators. We also give theoretical arguments why we expect this identification to be correct.

\subsection{Outline of contents}

In section 2, we recall basic facts related to Nekrasov's instanton partition functions. In section 3, we recall the AGT parametrisation of $\mathcal{M}^{\text {gen, } \mathcal{H}}$, the choice of parameters that allows us to obtain $\mathcal{M}^{p, p^{\prime}, \mathcal{H}}$, then show how the unrestricted instanton partition functions give the wrong answer in the case of $\mathcal{B}_{1}^{p, p^{\prime}, \mathcal{H}}$ on the torus. In section 4 , we use the requirement that the summands remain well-defined to characterise the partition pairs that label them. We identify these partition functions with $\mathcal{B}_{n}^{p, p^{\prime}, \mathcal{H}}$ In section 5 , we study the vanishing of the numerator, and show that whenever the denominator of a summand vanishes, then the numerator also vanishes. In section 6 , we check the correctness of our expressions in the two cases listed above. In section 7 , we use results from $[5,10,11]$, Proposition 4.1 


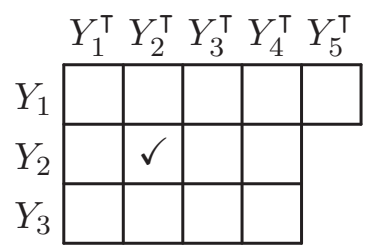

Figure 1. The Young diagram $Y$ of $5+4+4$. The rows are numbered from top to bottom. The rows of the transpose $Y^{\top}$ that represents $3+3+3+3+1$, which are the columns of $Y$, are numbered from left to right. From the viewpoint of $Y$, the marked cell $\nabla$ has $A_{\square, Y}=2, A_{\square, Y}^{+}=3$, and $L_{\square, Y}=1$. From the viewpoint of $Y^{\top}$, $\square$ has $A_{\square, Y \top}=1, A_{\square, Y \top}^{+}=2$, and $L_{\square, Y \top}=2$.

in section 4, and Conjecture 7.1 in section 7, to explain why the restriction to Burge pairs produces conformal blocks in $\mathcal{M}^{p, p^{\prime}, \mathcal{H}}$. Because we use Conjecture 7.1 , this explanation is not a proof. In section 8, we extend of our results to conformal blocks in $\mathcal{M}^{\text {gen,H }}$, with degenerate intermediate Virasoro representations, and in section 9, we collect a number of remarks that include 1. a conjectural generalization to the $W_{N}$ conformal blocks, and 2. a geometric interpretation of the summation over Burge pairs as a summation over isolated torus fixed points on the instanton moduli space.

\section{The instanton partition function}

\subsection{Partitions}

A partition $\pi$ of an integer $|\pi|$ is a set of non-negative integers $\left\{\pi_{1}, \pi_{2}, \cdots, \pi_{p}\right\}$, where $p$ is the number of parts, $\pi_{i} \geqslant \pi_{i+1}$, and $\sum_{i=1}^{p} \pi_{i}=|\pi| . \pi$ is represented as a Young diagram $Y$, which is a set of $p$ rows $\left\{Y_{1}, Y_{2}, \cdots, Y_{p}\right\}$, such that the $i$-th row has $Y_{i}=\pi_{i}$ cells, ${ }^{6} Y_{i} \geqslant Y_{i+1}$, and $|Y|=\sum_{i} Y_{i}=|\pi|$. We use $Y_{i}^{\top}$ for the transpose of $Y_{i}$, and define $Y_{i, \rho}^{+}=Y_{i, \rho}+1$.

We use $\square$ for a cell in a Young diagram $Y$, which is a square in the south-east quadrant of the plane, with coordinates $\{\rho, \sigma\}$, such that $\rho$ is the row-number, counted from top to bottom, and $\sigma$ is the column number, counted from left to right. We define $A_{\square, Y_{i}}^{+}=$ $A_{\square, Y_{i}}+1$, where $A_{\square, Y_{i}}$ is the arm of $\square$ in $Y_{i}$, that is, the number of cells in the same row as, but to the right of $\square$ in $Y_{i}$, and $L_{\square, W_{j}}$ to be the leg of $\square$ with respect its position in $W_{j}$, that is the number of cells in the same column as, but below $\square$ in $Y_{i}$.

\subsection{Partition pairs}

The AGT representation of $\mathcal{B}^{p, p^{\prime}, \mathcal{H}}$ involves a multi-sum over internal states labeled by $n+2$ partition pairs $\vec{Y}^{\iota}, \iota=0,1, \cdots, n, n+1$, where $\vec{Y}^{\iota}$ is a pair of Young diagrams, $\left\{Y_{1}^{\iota}, Y_{2}^{\iota}\right\}$, and $\left|\vec{Y}^{\iota}\right|=\left|Y_{1}^{\iota}\right|+\left|Y_{2}^{\iota}\right|$ is the total number of cells in $\vec{Y}^{\iota}$. The pairs $\left\{Y_{1}^{\iota}, Y_{2}^{\iota}\right\}$,

\footnotetext{
${ }^{6}$ We use $Y_{i}$ for the $i$-th row as well as for the number of cells in that row.
} 

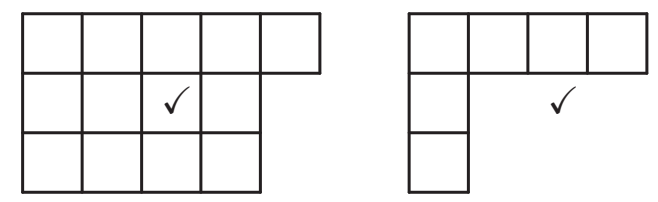

Figure 2. A partition pair $\left\{Y_{1}, Y_{2}\right\}$. $Y_{1}$ is on the left, $Y_{2}$ is on the right. The cell $\nabla$ has coordinates $(2,3), \nabla \in Y_{1}$, but $\nabla \notin Y_{2}$. It has $A_{\square, Y_{1}}=1, A_{\square, Y_{1}}^{+}=2, L_{\square, Y_{1}}=1$, as well as $A_{\square, Y_{2}}=-2$, $A_{\square, Y_{2}}^{+}=-1$, and $L_{\square, Y^{\top}}=-1$.

$\iota \in\{1, \cdots, n\}$, are non-empty Young diagrams, while $\left\{Y_{1}^{\iota}, Y_{2}^{\iota}\right\}, \iota \in\{0, n+1\}$ are empty, ${ }^{7}$ $\vec{Y}^{(0)}=\vec{Y}^{(n+1)}=\vec{\emptyset}$, where $\vec{\emptyset}$ is a pair of empty Young diagrams.

\subsection{A decomposition of the instanton partition function}

Consider the four-dimensional $\mathcal{N}=2$ supersymmetric linear quiver gauge theory with a gauge group $\prod_{\iota=1}^{n+1} \mathrm{U}(2)_{\iota}$, that is $(n+1)$ copies of $\mathrm{U}(2)$ [3]. The instanton partition function of this theory can be written in terms of 'building block' partition functions $Z_{b b}^{\iota}$, $\iota=1, \cdots, n+1$, as follows

$$
Z_{\mathrm{Nek}}=\sum_{\vec{Y}^{1}, \cdots, \vec{Y}^{n}} \prod_{\iota=1}^{n+1} q_{\iota}^{\left|\vec{Y}^{\iota}\right|} Z_{b b}^{\iota}\left(\vec{a}^{\iota-1}, \vec{Y}^{\iota-1}\left|\mu^{\iota}\right| \vec{a}^{\iota}, \vec{Y}^{\iota}\right)
$$

where $q_{\iota}$ is an indeterminate. In gauge theory, $q_{\iota}=e^{2 \pi i \tau_{\iota}}$, where $\tau_{\iota}$ is the complexified coupling constant of $\mathrm{U}(2)_{\iota}$. In conformal field theory, it is a rational function of the positions $z_{\iota}, \iota=0,1, \cdots, n+2$, of the vertex operators $\mathcal{O}_{\iota}$, whose expectation value is the conformal block, on the Riemann surface $\mathcal{S}$ that the conformal field theory is defined on. $Z_{b b}^{\iota}$ is defined in subsection 2.4 .

The decomposition of the instanton partition function in (2.1) follows that in [12] and mirrors the decomposition of conformal blocks on a sphere, represented as a comb diagram in figure 3.

\subsection{The building block of the instanton partition function}

$Z_{b b}$ is

$$
Z_{b b}(\vec{a}, \vec{Y}|\mu| \vec{b}, \vec{W})=\frac{z_{\text {num }}(\vec{a}, \vec{Y}|\mu| \vec{b}, \vec{W})}{z_{\operatorname{den}}(\vec{a}, \vec{Y} \mid \vec{b}, \vec{W})}
$$

The parameters that appear in $Z_{b b}$ are as follows.

\footnotetext{
${ }^{7}$ We work in terms of $n+2$ linearly-ordered partition pairs. Since we consider conformal blocks of primary fields, the initial and final pairs are always empty, but we prefer to work in terms of $n+2$ rather than $n$ non-empty pairs to make the notation in the sequel more uniform.
} 


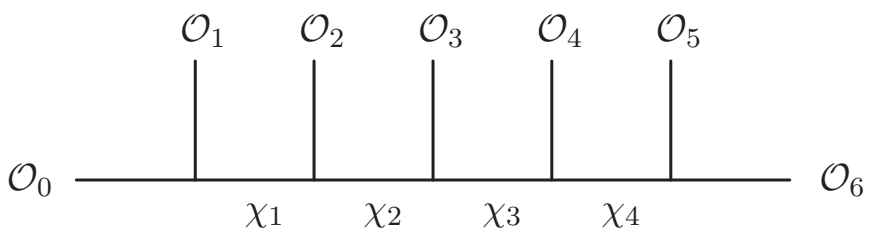

Figure 3. The comb diagram of a 4-channel conformal block that corresponds to a linear quiver. It consists of an initial state that corresponds to a vertex operator $\mathcal{O}_{0}$ on the left, five vertex operator insertions $\mathcal{O}_{1}, \cdots, \mathcal{O}_{5}$, and a final state that corresponds to a vertex operator $\mathcal{O}_{6}$ on the right. $\mathcal{O}_{\iota}$ is placed at $z_{\iota}$, where $z_{0}, z_{n+1}$ and $z_{n+2}$ are set 0,1 and $\infty$, respectively. In this example, $n=4$.

\subsubsection{The 2-component vector $\vec{a}^{\iota}=\left\{a^{\iota},-a^{\iota}\right\}$}

In gauge theory, $a^{\iota}$ is the expectation value of the vector multiplet in the adjoint representation of the gauge group $\mathrm{U}(2)_{\iota}$. In conformal field theory, $a^{\iota}$ is the charge of the highest weight of the Virasoro irrep that flows in channel $\chi_{\iota}$ in the conformal block under consideration.

\subsubsection{The partition pairs $\vec{Y}$ and $\vec{W}$}

In gauge theory, each partition pair $\vec{Y}^{\iota}=\left\{Y_{1}^{\iota}, Y_{2}^{\iota}\right\}$ labels the fixed localization points in the instanton moduli space of $\mathrm{U}(2)_{\iota}$. In conformal field theory, they label the states that flow in channel $\chi_{\iota}$ in the corresponding conformal block. In (2.2), $\vec{Y}$ and $\vec{W}$ are attached to the line segments on the left and the right of a given vertex, respectively.

\subsubsection{The scalar $\mu^{\iota}$}

In gauge theory, $\mu^{\iota}$ is the mass parameter of the bi-fundamental matter field that interpolates the gauge groups $\mathrm{U}(2)_{\iota}$ and $\mathrm{U}(2)_{\iota+1}$. In conformal field theory, $\mu^{\iota}$ is the charge of the vertex operator that connects channels $\chi_{\iota}$ and $\chi_{\iota+1}$. In the following, we study the structure of the right hand side of (2.2).

\subsubsection{The denominator}

where

$$
z_{\mathrm{den}}(\vec{a}, \vec{Y} \mid \vec{b}, \vec{W})=\left(z_{\mathrm{norm}}(\vec{a}, \vec{Y}) z_{\mathrm{norm}}(\vec{b}, \vec{W})\right)^{\frac{1}{2}}
$$

$$
z_{\text {norm }}(\vec{a}, \vec{Y})=z_{\text {num }}(\vec{a}, \vec{Y}|0| \vec{a}, \vec{Y})
$$

In gauge theory, $z_{\text {norm }}$ is a normalization factor related to the contribution of the vector multiplets that the bi-fundamental couples to. In conformal field theory, it accounts for the norms of the states that propagate into and out of the vertex operator insertion in $Z_{b b}$.

\subsubsection{The numerator}

$$
\begin{aligned}
& z_{\text {num }}(\vec{a}, \vec{Y}|\mu| \vec{b}, \vec{W})= \\
& \prod_{i, j=1}^{2} \prod_{\square \in Y_{i}}\left(E\left[a_{i}-b_{j}, Y_{i}, W_{j}, \square\right]-\mu\right) \prod_{\mathbf{\square} \in W_{j}}\left(\epsilon_{1}+\epsilon_{2}-E\left[b_{j}-a_{i}, W_{j}, Y_{i}, \mathbf{\square}\right]-\mu\right),
\end{aligned}
$$


where the elementary function $E\left[x, Y_{i}, W_{j}, \square\right]$ is defined as

$$
E\left[x, Y_{i}, W_{j}, \square\right]=x+A_{\square, Y_{i}}^{+} \epsilon_{2}-L_{\square, W_{j}} \epsilon_{1},
$$

$x$ is an indeterminate, and $\left\{\epsilon_{1}, \epsilon_{2}\right\}$ are Nekrasov's deformation parameters, which are generally complex. In gauge theory, $z_{\text {num }}$ is the contribution of a bi-fundamental multiplet in $\mathrm{U}(2)_{\iota}$ and $\mathrm{U}(2)_{\iota+1}$. In conformal field theory, it is the contribution of the vertex operator insertion that inputs a charge $\mu$ into the conformal block into $Z_{b b}$.

\subsubsection{Remark}

One can think of $z_{\text {num }}$ as the basic object in U(2) AGT theory and in this paper, and all other objects can be written in terms of special cases of it.

\subsubsection{Normalisation}

Consider the special case where the Virasoro part of the vertex operator in $Z_{b b}$ is the identity, that is $\{r, s\}=\{1,1\}$, and consequently $\mu=0 .{ }^{8} Z_{b b}$ is defined combinatorially and does not necessarily vanish when the fusion rules are not satisfied. To ensure that the fusion rules are satisfied, we set $\vec{a}=\vec{b}$.

Setting $\mu=0$ and $\vec{a}=\vec{b}$ ensures that the Virasoro part of the vertex operator insertion is the identity operator, but then, [5], the Heisenberg part of the vertex operator is an exponential of the creation part of the free boson field, which can contribute to a difference between $\vec{Y}$ and $\vec{W}$, and we do not necessarily have $\vec{Y}=\vec{W}$. Setting $\vec{Y}=\vec{W}$, we pick up the contribution of the trivial part of the exponential, that is the identity, and $Z_{b b}$ reduces to

$$
Z_{b b}(\vec{a}, \vec{Y}|0| \vec{a}, \vec{Y})=\frac{z_{\text {num }}(\vec{a}, \vec{Y}|0| \vec{a}, \vec{Y})}{z_{\operatorname{den}}(\vec{a}, \vec{Y} \mid \vec{a}, \vec{Y})}=1
$$

Equation (2.7) is relevant to computing 1-point conformal blocks of the identity operator on the torus in subsections 3.4 and 4.13 .

\section{Unrestricted instanton partition functions for $\mathcal{M}^{p, p^{\prime}, \mathcal{H}}$}

\subsection{AGT parameterisation. Generic models}

A generic model is a conformal field theory characterised by a central charge $c_{\text {gen }}$ that we parametrise as

$$
c_{\text {gen }}=1+6\left(b_{\text {gen }}+\frac{1}{b_{\text {gen }}}\right)^{2}, \quad b_{\text {gen }}=\left(\frac{\epsilon_{2}}{\epsilon_{1}}\right)^{\frac{1}{2}},
$$

In the Coulomb gas approach to computing conformal blocks in generic models, the screening charges $\left\{\beta_{+}, \beta_{-}\right\}$, and the background charge, $-2 \beta_{0}$, satisfy ${ }^{9}$

$$
\beta_{+}=b_{\text {gen }}, \quad \beta_{-}=\frac{1}{b_{\text {gen }}}, \quad 2 \beta_{0}=\beta_{+}+\beta_{-}
$$

\footnotetext{
${ }^{8}$ See subsection 5.2 .

${ }^{9}$ We use $\beta_{+}, \beta_{-},-2 \beta_{0}$ for generic model charges and reserve $\alpha_{+}, \alpha_{-}$and $-2 \alpha_{0}$ for the corresponding minimal model charges. We use $b_{\text {gen }}$ and $a_{p, p^{\prime}}$ for the parameters used to describe the generic and minimal models central charges respectively, since $a$ and $b$ are used for other purposes in the sequel.
} 


\subsection{AGT parameterisation. Minimal models}

A minimal model $\mathcal{M}^{p, p^{\prime}}$, based on a Virasoro algebra $\mathcal{V}^{p, p^{\prime}}$, characterised by a central charge $c_{p, p^{\prime}}<1$, that we parameterise as

$$
c_{p, p^{\prime}}=1-6\left(a_{p, p^{\prime}}-\frac{1}{a_{p, p^{\prime}}}\right)^{2}, \quad a_{p, p^{\prime}}=\left(\frac{p^{\prime}}{p}\right)^{\frac{1}{2}},
$$

where $\left\{p, p^{\prime}\right\}$ are the minimal model parameters, which are co-prime integers and satisfy $0<p<p^{\prime}$, in our conventions. In the Coulomb gas approach to computing conformal blocks in minimal models with $c<1[13,14]$, the screening charges $\left\{\alpha_{+}, \alpha_{-}\right\}$, and the background charge, $-2 \alpha_{0}$, satisfy

$$
\alpha_{+}=a_{p, p^{\prime}}, \quad \alpha_{-}=-\frac{1}{a_{p, p^{\prime}}}, \quad 2 \alpha_{0}=\alpha_{+}+\alpha_{-}
$$

The AGT parameterisation of $\mathcal{M}^{p, p^{\prime}, \mathcal{H}}$ is obtained by choosing

$$
\epsilon_{1}<0<\epsilon_{2}, \quad \epsilon_{1}=\alpha_{-}, \quad \epsilon_{2}=\alpha_{+}
$$

so that $\alpha_{-}<0<\alpha_{+}$. Since we focus on $\mathcal{M}^{p, p^{\prime}, \mathcal{H}}$, we work in terms of $\left\{\alpha_{-}, \alpha_{+}\right\}$instead of $\left\{\epsilon_{1}, \epsilon_{2}\right\}$, and write the elementary function $E\left[x, Y_{i}, W_{j}, \square\right]$ as

$$
E\left[x, Y_{i}, W_{j}, \square\right]=x+A_{\square, i}^{+} \alpha_{+}-L_{\square, j} \alpha_{-}
$$

\subsection{Charge content}

We need two distinct objects that, in Coulomb gas terms, are expressed in terms of the screening charges $\left\{\alpha_{+}, \alpha_{-}\right\}$. 1. The charge $\mu_{r, s}$ of the vertex operator $\mathcal{O}_{\mu}$ that intertwines two irrep's $\mathcal{H}_{r_{1}, s_{1}}^{p, p^{\prime}}$ and $\mathcal{H}_{r_{2}, s_{2}}^{p, p^{\prime}}$, and 2. The highest weight $\left|a_{r, s}\right\rangle$ of an irrep $\mathcal{H}_{r, s}^{p, p^{\prime}}$. Following [1, $5]$, we use $\{r, s\}$ as indices for the charge $\mu_{r, s}$ of the vertex operator $\mathcal{O}_{\mu_{r, s}}$, and $\{r, s\}$ as indices for the charge $a_{r, s}$ of the highest weight $\left|a_{r, s}\right\rangle$. These charges are parameterised in terms of $\alpha_{+}$and $\alpha_{-}$as follows

$$
\begin{aligned}
& \mu_{r, s}=-\left(\frac{r-1}{2}\right) \alpha_{+}-\left(\frac{s-1}{2}\right) \alpha_{-}, \\
& a_{r, s}=-\frac{r}{2} \alpha_{+}-\frac{s}{2} \alpha_{-}, \quad 1 \leqslant r \leqslant p-1,1 \leqslant s \leqslant p^{\prime}-1
\end{aligned}
$$

Note that the same numerical values of $\{r, s\}$ indicate different charge contents in $\mu_{r, s}$ and in $a_{r, s}$. In particular,

$$
2 \mu_{r, s}=2 a_{r, s}+\left(\alpha_{+}+\alpha_{-}\right)
$$

\subsection{Unrestricted instanton partition functions give incorrect 1-point functions on the torus}

Consider a conformal block in a 1-point function in $\mathcal{M}^{p, p^{\prime}, \mathcal{H}}$ on a torus, figure 4 .

Following [1], this is given by the instanton partition function of the $\mathcal{N}=2^{\star} \mathrm{U}(2)$ theory,

$$
Z_{\mathrm{Nek}}^{\mathcal{N}=2^{\star}, \mathrm{U}(2)}(\vec{a}, \vec{\mu})=\sum_{\vec{Y}} q^{|\vec{Y}|} \frac{z_{\mathrm{num}}(\vec{a}, \vec{Y}|\mu| \vec{a}, \vec{Y})}{z_{\mathrm{den}}(\vec{a}, \vec{Y} \mid \vec{a}, \vec{Y})},
$$




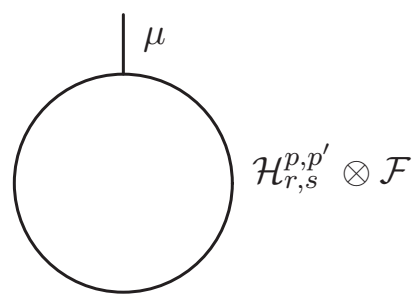

Figure 4. A graph of a conformal block of a 1-point function on a torus. The vertex operator insertion carries a charge $\mu$. The chiral states that flow in the channel belong to $\mathcal{H}_{r, s}^{p, p^{\prime}} \otimes \mathcal{F}$. For this 1-point function to be finite, the fusion rules must be satisfied.

where $\mu$ is determined by the operator insertion, and $\vec{a}$ is determined by the states of the $\mathcal{H}_{r, s}^{p, p^{\prime}}$ that flow in the torus and determine the conformal block. When the inserted operator is the identity, that is $\{r, s\}$, then ${ }^{10} \mu=0$, and if $\vec{Y}$ is an unrestricted partition pair as in the original AGT prescription, then

$$
Z_{\mathrm{Nek}}^{\mathcal{N} 2^{\star}, \mathrm{U}(2)}(\vec{a}, \overrightarrow{0})=\sum_{\vec{Y}} q^{|\vec{Y}|}=\frac{1}{\prod_{n=1}^{\infty}\left(1-q^{n}\right)^{2}}
$$

while the correct result is

$$
Z_{\mathrm{Nek}}^{\mathcal{N} 2^{\star}, \mathrm{U}(2)}(\vec{a}, \overrightarrow{0})=\frac{\chi_{r, s}^{p, p^{\prime}}}{\prod_{n=1}^{\infty}\left(1-q^{n}\right)},
$$

where $\chi_{r, s}^{p, p^{\prime}}$ is the character of the irrep $\mathcal{H}_{r, s}^{p, p^{\prime}}$ that flows in the torus, and $\vec{a}=\{a,-a\}$, where $2 a=-r \alpha_{+}-s \alpha_{-}$,

$$
\chi_{r, s}^{p, p^{\prime}}=\frac{\sum_{k=-\infty}^{\infty}\left(q^{k^{2} p p^{\prime}+k\left(p^{\prime} r-p s\right)}-q^{(k p+r)\left(k p^{\prime}+s\right)}\right)}{\prod_{n=1}^{\infty}\left(1-q^{n}\right)}
$$

This simple example makes it clear that applying the prescription of [1] to $\mathcal{M}^{p, p^{\prime}, \mathcal{H}}$ without modification, leads to incorrect answers. In the following section, we find that it leads to zeros in the denominators of the summands.

\section{Restricted instanton partition functions for $\mathcal{M}^{p, p^{\prime}, \mathcal{H}}$. The denominator}

Consider the denominator $z_{\text {den }}$ of $Z_{b b}$ in (2.2). To look for zeros in $z_{\text {den }}$, it is sufficient to look for zeros in $z_{\text {norm }}[\vec{a}, \vec{Y}]$ in $(2.4)$. Consider $\mathcal{B}_{n}^{p, p^{\prime}, \mathcal{H}}$ and focus on a channel that carries states that belong to $\mathcal{H}_{r, s}^{p, p^{\prime}}$.

Proposition $4.1 z_{\text {norm }}[\vec{a}, \vec{Y}] \neq 0$, if and only if

$$
Y_{2, \sigma}^{\top}-Y_{1, \sigma+r-1}^{\top} \geqslant 1-s, \quad Y_{1, \sigma}^{\top}-Y_{2, \sigma+[p-r]-1}^{\top} \geqslant 1-\left(p^{\prime}-s\right),
$$

where $Y_{i, \sigma}^{\top}$ is the $\sigma$-column in $Y_{i}, i \in\{1,2\}$.

The proof of Proposition 4.1 is based on checking the products that appear in $z_{\text {norm }}[\vec{a}, \vec{Y}]$ for zeros.

\footnotetext{
${ }^{10}$ See subsection 5.2 .
} 


\subsection{More notation}

We set $a_{1}=-a_{2}=a$, and $a_{1}-a_{2}=2 a$. If a channel $\chi_{\iota}$ carries states from $\mathcal{H}_{r_{\iota}, s_{\iota}}^{p, p^{\prime}} \otimes \mathcal{F}$, then the label $a_{\iota}$ of the corresponding highest weight is

$$
a_{r_{\iota}, s_{\iota}}=-r_{\iota} \alpha_{+}-s_{\iota} \alpha_{-}
$$

\subsection{Two zero-conditions}

In the sequel, we find that an instanton partition function has a zero when an equation of type

$$
C_{+} \alpha_{+}+C_{-} \alpha_{-}=0
$$

where $\alpha_{-}<0<\alpha_{+}$, is satisfied. Equivalently, an instanton partition function has a zero when the two conditions

$$
C_{+}=c p, \quad C_{-}=c p^{\prime}
$$

are satisfied, where $c$ is some constant that needs to be determined.

\subsection{From two zero-conditions to one zero-condition}

Given two conditions, such as (4.4), we need, for the purposes of comparing with known results, to re-write them as one condition. Consider the two conditions

$$
A_{\square, 1}=A^{\prime} \geqslant 0, \quad-L_{\square, 2}=L^{\prime} \geqslant 0
$$

These conditions are satisfied if $\square \in Y_{1}$, and $\square \notin Y_{2} \cdot{ }^{11}$ If $\square$ is in row- $\rho$ and column- $\sigma$ in $Y_{1}$, then the first condition in (4.5) implies that there is a cell $\boxplus \in Y_{1}$, to the right of $\square$, with coordinates $\left\{\rho, \sigma+A^{\prime}\right\}$, that lies on a vertical boundary. In other words, $\mathbf{1}$. there are no cells to the right of $\boxplus$, and $\mathbf{2}$. there may or may not be cells below $\boxplus$. This means that the $\left(\sigma+A^{\prime}\right)$-column in $Y_{1}$, or equivalently, the $\left(\sigma+A^{\prime}\right)$-row in $Y_{1}^{\top}$, has length at least $\rho$,

$$
Y_{1, \sigma+A^{\prime}}^{\top} \geqslant \rho
$$

This allows us to write the second condition in (4.5) as

$$
-L_{\square, 2}=L^{\prime}=\rho-Y_{2, \sigma}^{\top},
$$

where the second equality in (4.7) follows from the definition of $L_{\square, 2}$. In other words, $\rho=L^{\prime}+Y_{2, \sigma}^{\top}$, and from (4.6),

$$
Y_{1, \sigma+A^{\prime}}^{\top}-Y_{2, \sigma}^{\top} \geqslant L^{\prime}
$$

which is one condition that is equivalent to the two conditions in (4.5).

\footnotetext{
${ }^{11}$ We chose the labels of the Young diagrams to be concrete. The same arguments apply under $Y_{1} \Leftrightarrow Y_{2}$.
} 


\subsection{One non-zero condition}

Consider a function $z\left[Y_{1}, Y_{2}\right]$, of a pair of Young diagrams $\left\{Y_{1}, Y_{2}\right\}$, such that $z\left[Y_{1}, Y_{2}\right]=0$, if and only if (4.8) is satisfied. This implies that $z\left[Y_{1}, Y_{2}\right] \neq 0$, if and only if $\left\{Y_{1}, Y_{2}\right\}$ satisfies the complementary condition

$$
Y_{1, \sigma+A^{\prime}}^{\top}-Y_{2, \sigma}^{\top}<L^{\prime}
$$

which, in order to compare with results in the literature, we choose to write as

$$
Y_{2, \sigma}^{\top}-Y_{1, \sigma+A^{\prime}}^{\top} \geqslant 1-L^{\prime}
$$

\subsubsection{Remark}

Since we use equations such as (4.5) and (4.10) frequently in the sequel, refer to the former as 'zero-conditions', and to the latter as 'non-zero-conditions'.

\subsection{Products that appear in the denominator}

Two types of products appear in $z_{\text {norm }}, \mathbf{1}$. products in the form $\prod_{\square \in Y_{i}} E\left[a_{i}-a_{j}, Y_{i}, Y_{j}, \square\right]$ that we refer to as $\left\{Y_{i}, Y_{j}\right\}_{\text {den }}$, and 2. products in the form $\prod_{\square \in Y_{i}}\left[\alpha_{+}+\alpha_{-}-E\left[a_{i}-\right.\right.$ $\left.\left.a_{j}, Y_{i}, Y_{j}, \square\right]\right]$ that we refer to as $\left\{Y_{i}, Y_{j}\right\}_{\text {den }}^{\prime}$.

\subsection{In search of zeros}

In the following subsections, $\mathbf{1}$. we consider the products that appear in $z_{\text {den }}$, one at a time, 2. we search for possible zeros, as in subsection 4.2, 3. we find the conditions that we need to impose on the pair $\left\{Y_{1}, Y_{2}\right\}$ in order to avoid the zeros, and 4 . when there is more than one set of conditions to avoid the zeros, we choose the stronger set. That is, the set that ensures that all zeros are eliminated. We use the fact that $r, s, p-a$ and $p^{\prime}-s$ are non-zero positive integers.

\section{7 $\left\{Y_{1}, Y_{1}\right\}_{\text {den }}$}

This product does not vanish, since this requires that there is a factor that satisfies

$$
E\left[0, Y_{1}, Y_{1}, \square\right]=A_{\square, 1}^{+} \alpha_{+}-L_{\square, 1} \alpha_{-}=0,
$$

which is not possible since $\square \in Y_{1}$ and $\alpha_{-}<0$.

\section{$4.8\left\{Y_{1}, Y_{1}\right\}^{\prime},\left\{Y_{2}, Y_{2}\right\}_{\text {den }}$ and $\left\{Y_{2}, Y_{2}\right\}_{\text {den }}^{\prime}$}

These products do not vanish for the same reason that $\left\{Y_{1}, Y_{1}\right\}_{\text {den }}$ in paragraph 4.7 does not vanish.

\section{$4.9\left\{Y_{1}, Y_{2}\right\}_{\text {den }}$}

This product vanishes if any factor satisfies

$$
E\left[-r \alpha_{+}-s \alpha_{-}, Y_{1}, Y_{2}, \square\right]=\left(-r+A_{\square, 1}^{+}\right) \alpha_{+}+\left(-s-L_{\square, 2}\right) \alpha_{-}=0,
$$


which lead to the conditions

$$
A_{\square, 1}=r-1+c p \quad-L_{\square, 2}=s+c p^{\prime}
$$

Since $A_{\square, i}, L_{\square, i}, i \in\{1,2\}, r$, and $s$ are non-zero positive integers, and $p$ and $p^{\prime}$ are positive co-primes, $c$ must be a non-negative integer, and the conditions in (4.13) are possible for $c=\{0,1, \cdots\}, \square \in Y_{1}$ and $\square \notin Y_{2}{ }^{12}$

\subsubsection{From two zero-conditions to one non-zero-condition}

Following paragraphs 4.3 and 4.4, the two zero-conditions in (4.13) can be translated to one non-zero-condition,

$$
Y_{1, \sigma}^{\top}-Y_{2, \sigma+r-1+c p}^{\top} \geqslant 1-s-c p^{\prime}
$$

\subsubsection{The stronger condition}

Equation (4.14) is the statement that to eliminate the zeros, we want $Y_{2, \sigma}^{\top}-Y_{1, \sigma+r-1+c p}^{\top} \geqslant$ $1-s-c p^{\prime}$, where $c=\{0,1, \cdots\}$ Since the row-lengths of a partition are by definition weakly decreasing, and $c=\{0,1, \cdots\}$, this is the case if $Y_{2, \sigma}^{\top}-Y_{1, \sigma+r-1}^{\top} \geqslant 1-s-c p^{\prime}$, which is the case if $Y_{2, \sigma}^{\top}-Y_{1, \sigma+r-1}^{\top} \geqslant 1-s$. Thus, we should set $c=0$, and obtain

$$
Y_{2, \sigma}^{\top}-Y_{1, \sigma+r-1}^{\top} \geqslant 1-s
$$

\section{$4.10\left\{Y_{2}, Y_{1}\right\}_{\text {den }}$}

This product vanishes if any factor satisfies

$$
E\left[r \alpha_{+}+s \alpha_{-}, Y_{2}, Y_{1}, \square\right]=\left(r+A_{\square, 2}^{+}\right) \alpha_{+}+\left(s-L_{\square, 1}\right) \alpha_{-}=0,
$$

which leads to the conditions

$$
A_{\square, 2}=-1-r+c p, \quad-L_{\square, 1}=-s+c p^{\prime},
$$

which are possible for $c=\{1,2, \cdots\}, \square \in Y_{2}$ and $\square \notin Y_{1}$.

\subsubsection{From two zero-conditions to one non-zero-condition}

Following paragraphs 4.3 and 4.4, the two zero-conditions in (4.17) can be translated to one non-zero-condition,

$$
Y_{1, \sigma}^{\top}-Y_{2, \sigma-1-r+c p}^{\top} \geqslant 1+s-c p^{\prime}
$$

\subsubsection{The stronger condition}

Equation (4.18) is the statement that to eliminate the zeros, we want $Y_{2, \sigma}^{\top}-Y_{1, \sigma-1-r+c p}^{\top} \geqslant$ $1+s-c p^{\prime}$, where $c=\{1,2, \cdots\}$. Since the row-lengths of a partition are by definition weakly decreasing, and $c=\{1,2, \cdots\}$, this is the case if $Y_{2, \sigma}^{\top}-Y_{1, \sigma+p-r-1}^{\top} \geqslant 1-s-c p^{\prime}$, which is the case if $Y_{2, \sigma}^{\top}-Y_{1, \sigma+p-r-1}^{\top} \geqslant 1-p+s$. Thus, we should set $c=1$, to obtain

$$
Y_{1, \sigma}^{\top}-Y_{2, \sigma+[p-r]-1}^{\top} \geqslant 1-\left(p^{\prime}-s\right)
$$

\footnotetext{
${ }^{12}$ Note that from conditions (4.13), if the Young diagram $Y$ such that $\square \in Y$, which in this case is $Y_{1}$, is sufficiently large compared to the Young diagram $W$ such that $\square \notin W$, which in this case is $Y_{2}$, then the product under discussion will have more than one zero. This will be the case in the rest of the factors discussed in this section as well.
} 


\section{$4.11\left\{Y_{1}, Y_{2}\right\}_{\text {den }}^{\prime}$}

This product vanishes if any factor satisfies

$$
\begin{aligned}
-\alpha_{+}-\alpha_{-}+E\left[-r \alpha_{+}-s \alpha_{-}, Y_{1}, Y_{2}, \square\right] & = \\
\left(-r+A_{\square, 1}\right) \alpha_{+}+\left(-s-L_{\square, 2}^{+}\right) \alpha_{-} & =0,
\end{aligned}
$$

which leads to the conditions

$$
A_{\square, 1}=r+c p, \quad-L_{\square, 2}=1+s+c p^{\prime},
$$

which, using the same arguments as in subsections 4.9 and 4.10, are possible for $c=$ $\{0,1, \cdots\}, \square \in Y_{1}, \square \notin Y_{2}$, and we should choose $c=0$ to obtain

$$
Y_{2, \sigma}^{\top}-Y_{1, \sigma+r}^{\top} \geqslant-s
$$

Comparing condition (4.15) with condition (4.22), we see that the former is stronger than the latter, for the same reasons as in paragraph 4.9.2. Thus this case does not offer new conditions on the partition pair.

\section{$4.12\left\{Y_{2}, Y_{1}\right\}_{\text {den }}^{\prime}$}

This product vanishes if any factor satisfies

$$
\begin{aligned}
-\alpha_{+}-\alpha_{-}+E\left[r \alpha_{+}+s \alpha_{-}, Y_{2}, Y_{1}, \square\right] & = \\
\left(r+A_{\square, 2}\right) \alpha_{+}+\left(s-L_{\square, 1}^{+}\right) \alpha_{-} & =0,
\end{aligned}
$$

which leads to the conditions

$$
A_{\square, 2}=-r+c p, \quad-L_{\square, 1}=1-s+c p^{\prime},
$$

which, using the same arguments as in subsections 4.9 and 4.10 , are possible for $c=$ $\{1,2, \cdots\}, \square \in Y_{2}$ and $\square \notin Y_{1}$, and we should choose $c=1$ to obtain

$$
Y_{2, \sigma}^{\top}-Y_{1, \sigma+[p-r]}^{\top} \geqslant-\left(p^{\prime}-s\right)
$$

Comparing condition (4.19) with condition (4.25), we see that the former is stronger than the latter, for the same reasons as in paragraph 4.10.2. Thus this case does not offer new conditions on the partition pair.

\subsection{Restricted instanton partition functions give the correct 1-point function on the torus}

From the discussion in paragraphs $4.7-4.12$, we conclude that $z_{\text {den }}$ has no zeros if the conditions in (4.15) and (4.19) are satisfied. As mentioned in section 1, these conditions on partition pairs are known. They were introduced and studied in [9], and were further studied and called Burge pairs in [10]. A full and explicit derivation of the fact that the generating function of the Burge pairs, that satisfy conditions (4.15) and (4.19), is the $q$-series in (3.11), we refer the reader to appendix A of [10]. 


\subsubsection{Remark}

The conditions obtained in this note were written differently in $[9,10]$ for three reasons. 1. These papers used the notation $\{a, b, \alpha, \beta\}$, which in terms of the variables $\left\{r, s, p, p^{\prime}\right\}$ used in this work are $a=r, b=p-r, \alpha=s$, and $\beta=p^{\prime}-s, \mathbf{2}$. The partition rows were labeled such that $Y_{i} \geqslant Y_{i-1}$, while in this note, we assume the opposite (and more conventional) labeling, and 3. The conventions in $[9,10]$ are such that the conditions were expressed in terms of the Young diagrams that the presentation naturally started with, while in this work, we wished to follow the conventions of $[1,3,5]$, so we ended up expressing the conditions on the partition pair $\left\{Y_{1}, Y_{2}\right\}$ as conditions on $\left\{Y_{1}^{\top}, Y_{2}^{\top}\right\}$.

\section{Restricted instanton partition functions for $\mathcal{M}^{p, p^{\prime}, \mathcal{H}}$. The numerator}

\subsection{Products that appear in the numerator}

Two types of products appear in $z_{\text {num }}, \mathbf{1}$. products in the form $\prod_{\square \in Y_{i}}\left[E\left[x, Y_{i}, W_{j}, \square\right]-\mu\right]$ that we refer to as $\left\{Y_{i}, W_{j}\right\}_{\text {num }}$, and $\mathbf{2}$. products in the form $\prod_{\square \in W_{j}}\left[\alpha_{+}+\alpha_{-}-\right.$ $\left.E\left[-x, W_{j}, Y_{i}, \square\right]-\mu\right]$ that we refer to as $\left\{W_{j}, Y_{i}\right\}_{\text {num }}^{\prime}$. We need to examine the conditions that each of these factors imposes on the partition pairs $\left\{Y_{1}, Y_{2}\right\}$ and $\left\{W_{1}, W_{2}\right\}$.

\subsection{Notation}

We set

$$
\begin{gathered}
a_{1}=-a_{2}=a=-\left(\frac{m_{a}+1}{2}\right) \alpha_{+}-\left(\frac{n_{a}+1}{2}\right) \alpha_{-}, \\
m_{a} \in\{0,1, \cdots, p-2\}, n_{a} \in\left\{0,1, \cdots, p^{\prime}-2\right\} \\
b_{1}=-b_{2}=b=-\left(\frac{m_{b}+1}{2}\right) \alpha_{+}-\left(\frac{n_{b}+1}{2}\right) \alpha_{-}, \\
m_{b} \in\{0,1, \cdots, p-2\}, n_{b} \in\left\{0,1, \cdots, p^{\prime}-2\right\}, \\
\mu=-\frac{1}{2} m_{\mu} \alpha_{+}-\frac{1}{2} n_{\mu} \alpha_{-}, \quad m_{\mu} \in\{0,1, \cdots, p-2\}, n_{\mu} \in\left\{0,1, \cdots, p^{\prime}-2\right\},
\end{gathered}
$$

and use the subscript $a(b)$ to indicate the parameters that appear in the conditions on the partition pairs $\vec{Y}(\vec{W})$ that label the states in the incoming (outgoing) channel that flows towards (away from) the vertex operator insertion. It is useful to note that, in this notation,

$$
r_{a}=m_{a}+1, \quad s_{a}=n_{a}+1, \quad r_{b}=m_{a}+1, \quad s_{b}=n_{a}+1
$$

Further, to simplify the presentation, we use the notation

$$
M_{[ \pm, \pm, \pm]}=\frac{1}{2}\left( \pm m_{a} \pm m_{b} \pm m_{\mu}\right), \quad N_{[ \pm, \pm, \pm]}=\frac{1}{2}\left( \pm n_{a} \pm n_{b} \pm n_{\mu}\right)
$$

\subsection{The fusion rules}

In the notation of subsection 5.2, the fusion rules are

$$
m_{a}+m_{b}+m_{\mu}=0 \bmod 2, \quad n_{a}+n_{b}+n_{\mu}=0 \bmod 2
$$


and the triple $\left\{m_{a}, m_{b}, m_{\mu}\right\}$ satisfies the triangular conditions

$$
m_{a}+m_{b} \geqslant m_{\mu}, \quad m_{b}+m_{\mu} \geqslant m_{a}, \quad m_{\mu}+m_{a} \geqslant m_{b}
$$

with analogous triangular conditions for $\left\{n_{a}, n_{b}, n_{\mu}\right\}$.

\subsection{Bounds on $M_{[ \pm, \pm, \pm]}$and $\boldsymbol{N}_{[ \pm, \pm, \pm]}$}

For the purposes of the proofs in subsections 5.7 and 5.8, we need to show that $M_{[ \pm, \pm, \pm]}$ and $N_{[ \pm, \pm, \pm]}$satisfy the bounds

$$
0 \leqslant M_{[ \pm, \pm, \pm]} \leqslant p-2, \quad 0 \leqslant N_{[ \pm, \pm, \pm]} \leqslant p^{\prime}-2
$$

The lower bounds follow from the lower bounds in the definitions (5.1)-(5.3). The upper bounds are obtained as follows. There are two ways to choose the charge content of the highest weight state of a Virasoro irrep. The first choice is $\alpha_{r, s}$, where

$$
\begin{aligned}
2 \alpha_{r, s} & =-(r-1) \alpha_{+}-(s-1) \alpha_{-}, \\
r & =m+1, \quad s=n+1, \\
0 & \leqslant m \leqslant p-2, \quad 0 \leqslant n \leqslant p^{\prime}-2,
\end{aligned}
$$

while the second choice is

$$
\begin{aligned}
2 \alpha_{r^{\prime}, s^{\prime}} & =-\left(r^{\prime}-1\right) \alpha_{+}-\left(s^{\prime}-1\right) \alpha_{-}, \\
r^{\prime} & =m^{\prime}+1, \quad s^{\prime}=n^{\prime}+1, \\
0 & \leqslant m^{\prime} \leqslant p-2, \quad 0 \leqslant n^{\prime} \leqslant p^{\prime}-2,
\end{aligned}
$$

and the two choice are related by

$$
r^{\prime}=p-r, \quad s^{\prime}=p^{\prime}-s
$$

While the two representations are the same, for the purposes of the proofs in the sequel, we need to use one or the other, as follows.

Scanning a linear conformal block $\mathcal{B}_{n}^{p, p^{\prime}, \mathcal{H}}$ from left to right, one considers the building block $Z_{b b}^{\iota}, \iota=1,2, \cdots$, with the Virasoro irrep labeled by $\left\{r_{\iota-1}, s_{\iota-1}\right\}$ flowing in from the left, the vertex operator $\mathcal{O}_{\iota}$ of the primary field labeled by $\left\{r_{\mu}, s_{\mu}\right\}$ in the middle, and the Virasoro irrep labeled by $\left\{r_{\iota}, s_{\iota}\right\}$ flowing out to the right. Suppose that the charge content of the incoming primary field in $\chi_{\iota-1}$ is fixed. ${ }^{13}$ The charge content of primary state $\mu$ of the vertex operator $\mathcal{O}_{\mu}$ in the middle, and that of the outgoing primary field in $\chi_{\iota}$ are not fixed yet, and each can be chosen in one of two equivalent ways. We wish to show that we can choose these charge contents in such a way that that the upper bounds in (5.8) are satisfied. This will simplify our proofs in the sequel.

\footnotetext{
${ }^{13}$ Starting from $Z_{b b}^{1}$, we can choose the charge of the highest weight state in $\mathcal{O}_{0}$ either way, but for the purposes of this proof, it is sufficient to consider an arbitrary $Z_{b b}^{\iota}, \iota=1,2, \cdots, n+1$, and take the charge of the primary field in $\chi_{\iota-1}$ to be fixed.
} 
If $\left\{m_{\iota}, n_{\iota}\right\}$ and $\left\{m_{\mu}, n_{\mu}\right\}$ are such that the upper bounds in (5.8) are satisfied, then use this choice. If $\left\{m_{\iota}, n_{\iota}\right\}$ and $\left\{m_{\mu}, n_{\mu}\right\}$ are such that the upper bounds in (5.8) are not satisfied, we choose the dual representation of the vertex operator in the middle and the outgoing Virasoro irrep. ${ }^{14}$ In other words, $p-\frac{1}{2}\left(m_{\iota-1}+m_{\mu}+m_{\iota}\right)-2$, that does not satisfy the upper bound, becomes

$$
\begin{aligned}
& p-\frac{1}{2}\left(m_{\iota-1}+m_{\mu}^{\prime}+m_{\iota}^{\prime}\right)-2= \\
& p-\frac{1}{2}\left(m_{\iota-1}+p-m_{\mu}-2+p-m_{\iota}-2\right)-2= \\
& \frac{1}{2}\left(m_{\iota-1}+m_{\mu}+m_{\iota}\right) \geqslant 0
\end{aligned}
$$

using the triangular conditions (5.7), and similarly

$$
p^{\prime}-\frac{1}{2}\left(n_{\iota-1}+n_{\mu}^{\prime}+n_{\iota}^{\prime}\right)-2 \geqslant 0
$$

Now the charge content of the outgoing primary field is fixed and goes on to become the incoming primary field of $Z_{b b}^{\iota+1}$ or the primary state of $\mathcal{O}_{n+3}$. Thus we can always choose the charge contents such that the upper bounds in equations (5.12) and (5.13).

In the following subsections, we consider the conditions that products in the numerator must satisfy to be non-zero. ${ }^{15}$

\section{$5.5\left\{Y_{1}, W_{1}\right\}_{\text {num }}$}

This product vanishes if any factor satisfies

$$
\begin{aligned}
E\left[a-b, Y_{1}, W_{1}, \square\right]-\mu & = \\
-M_{[+,-,-]} \alpha_{+}-N_{[+,-,-]} \alpha_{-}+A_{Y_{1}}^{+} \alpha_{+}-L_{W_{1}} \alpha_{-} & =0,
\end{aligned}
$$

which leads to the zero-conditions

$$
A_{\square, Y_{1}}=-M_{[-,+,+]}+c p-1, \quad-L_{\square, W_{1}}=-N_{[-,+,+]}+c p^{\prime}
$$

From the triangular conditions (5.7), the maximal value of $M_{[-,+,+]}$is $p-2$, and the maximal value of $N_{[-,+,+]}$is $p^{\prime}-2$, thus the stronger condition corresponds to $c=1$, and we obtain two zero-conditions that we can write as one non-zero-condition,

$$
W_{1, \sigma}^{\top}-Y_{1, \sigma+\left[p-M_{[-,+,+]}-1\right]}^{\top} \geqslant-\left(p^{\prime}-N_{[-,+,+]}-1\right)
$$

\footnotetext{
${ }^{14}$ Remember that the charge content of the incoming primary field is given and cannot be changed.

${ }^{15}$ In writing equations (5.16), (5.19), and (5.20)-(5.24), we choose to group terms together in such a way to make the analogy with equations (4.15)-(4.25), that involve $\left\{Y_{1}, Y_{2}\right\}$ only, relatively more clear. Basically, $M$ and $N$ in the former are analogues of $(r-1)$ and $(s-1)$ in the latter.
} 


\section{$5.6\left\{Y_{1}, W_{1}\right\}_{\text {num }}^{\prime}$}

This product vanishes if any factor satisfies

$$
\begin{aligned}
E\left[-a+b, W_{1}, Y_{1}, \square\right]+\mu-2 \alpha_{0} & = \\
-\frac{1}{2}\left(-m_{a}+m_{b}+m_{\mu}\right) \alpha_{+}-\frac{1}{2}\left(-n_{a}+n_{b}+n_{\mu}\right) \alpha_{-}+A_{W_{1}} \alpha_{+}-L_{Y_{1}}^{+} \alpha_{-} & =0,
\end{aligned}
$$

which leads to the zero-conditions

$$
A_{\square, W_{1}}=M_{[-,+,+]}+c p, \quad-L_{\square, Y_{1}}=N_{[-,+,+]}+1+c p^{\prime}
$$

Following 5.5, we should set $c=0$, and (5.18) translates to the non-zero-condition

$$
Y_{1, \sigma}^{\top}-W_{1, \sigma+M_{[-,+,+]}^{\top}}^{\top} \geqslant-N_{[-,+,+]}
$$

\subsection{The remaining six products}

The analysis of the remaining six products is identical to that in 5.5 and 5.6, and it suffices to list the non-zero-condition in each case.

5.7.1 $\left\{Y_{2}, W_{2}\right\}_{\text {num }}$

$$
W_{2, \sigma}^{\top}-Y_{2, \sigma+\left[p-M_{[+,-,+]}-1\right]}^{\top} \geqslant-\left(p^{\prime}-N_{[+,-,+]}-1\right)
$$

\subsection{2 $\left\{Y_{2}, W_{2}\right\}_{\text {num }}^{\prime}$}

$$
Y_{2, \sigma}^{\top}-W_{2, \sigma+M_{[+,-,+]}}^{\top} \geqslant-N_{[+,-,+]}
$$

5.7.3 $\left\{Y_{1}, W_{2}\right\}_{\text {num }}$

$$
W_{2, \sigma}^{\top}-Y_{1, \sigma+M_{[+,+,-]}^{\top}}^{\top} \geqslant-N_{[+,+,-]}
$$

5.7.4 $\left\{Y_{1}, W_{2}\right\}_{\text {num }}^{\prime}$

$$
Y_{1, \sigma}^{\top}-W_{2, \sigma+\left[p-M_{[+,+,-]}-1\right]}^{\top} \geqslant-\left(p^{\prime}-N_{[+,+,-]}-1\right)
$$

5.7.5 $\left\{Y_{2}, W_{1}\right\}_{\text {num }}$

$$
W_{1, \sigma}^{\top}-Y_{2, \sigma+\left[p-M_{[+,+,+]}-1\right]-1}^{\top} \geqslant 1-\left(p^{\prime}-N_{[+,+,+]}-1\right)
$$

\subsection{6 $\left\{Y_{2}, W_{1}\right\}_{\text {num }}^{\prime}$}

$$
Y_{2, \sigma}^{\top}-W_{1, \sigma+M_{[+,+,+]}+1}^{\top} \geqslant-\left(N_{[+,+,+]}+1\right)
$$

\subsubsection{Remark}

Equations (5.23) and (5.24) make sense as Burge-type conditions because of the bounds in (5.12) and (5.13).

\subsection{If the denominator is zero, then the numerator is zero}

The non-zero-conditions on the $\left\{Y_{i}, W_{j}\right\}_{\text {num }}$ and $\left\{Y_{i}, W_{j}\right\}_{\text {num }}^{\prime}$ products that appear in the numerator can be combined in pairs to produce non-zero-conditions on $\left\{Y_{i}, Y_{j}\right\}$ and $\left\{W_{i}, W_{j}\right\}$ pairs, $i \neq j$ pairs also in the numerator, that can be compared to the first and second Burge non-zero-conditions (4.15) and (4.19) obtained from the denominator. 


\subsection{1 $\left\{Y_{1}, W_{1}\right\}_{\text {num }}$ and $\left\{Y_{2}, W_{1}\right\}_{\text {num }}^{\prime}$}

Consider conditions (5.16) and (5.25). We eliminate $W_{1}^{\top}$ to obtain a non-zero condition on $Y_{1}$ and $Y_{2}$ by re-writing (5.16) as

$$
W_{1, \sigma+\left[M_{[+,+,+]}+1\right]}^{\top}-Y_{1, \sigma+\left[p-M_{[-,+,+]}-1\right]+\left[M_{[+,+,+]}+1\right]}^{\top} \geqslant-p^{\prime}+N_{[-,+,+]}+1
$$

which combined with condition (5.25) gives

$$
Y_{2, \sigma}^{\top}-Y_{1, \sigma+\left[r_{a}-1\right]+p}^{\top} \geqslant 1-s_{a}-p^{\prime}
$$

which is a weak version of the first Burge condition (4.15).

\subsection{2 $\left\{Y_{1}, W_{2}\right\}_{\text {num }}$ and $\left\{Y_{2}, W_{2}\right\}_{\text {num }}^{\prime}$}

From (5.22) and (5.21),

$$
Y_{2, \sigma}^{\top}-Y_{1, \sigma+\left[r_{a}-1\right]}^{\top} \geqslant 1-s_{a}
$$

which is the first Burge condition (4.15).

\subsection{3 $\left\{Y_{2}, W_{1}\right\}_{\text {num }}$ and $\left\{Y_{1}, W_{1}\right\}_{\text {num }}^{\prime}$}

From (5.24) and (5.19),

$$
Y_{1, \sigma}^{\top}-Y_{2, \sigma+\left[p-r_{a}\right]-1}^{\top} \geqslant 1-\left[p^{\prime}-s_{a}\right],
$$

which is the second Burge condition (4.19).

\subsection{4 $\left\{Y_{2}, W_{2}\right\}_{\text {num }}$ and $\left\{Y_{1}, W_{2}\right\}_{\text {num }}^{\prime}$}

From (5.20) and (5.23),

$$
Y_{1, \sigma}^{\top}-Y_{2, \sigma+\left[p-r_{a}-1\right]+p}^{\top} \geqslant 1-\left[p^{\prime}-s_{a}\right]-p^{\prime},
$$

which is a weak version of the second Burge condition (4.19).

\subsection{5 $\left\{Y_{1}, W_{1}\right\}_{\text {num }}^{\prime}$ and $\left\{Y_{1}, W_{2}\right\}_{\text {num }}$}

From (5.19) and (5.22),

$$
W_{2, \sigma}^{\top}-W_{1, \sigma+r_{b}-1}^{\top} \geqslant 1-s_{b},
$$

which is the first Burge condition (4.15).

\subsection{6 $\left\{Y_{2}, W_{1}\right\}_{\text {num }}^{\prime}$ and $\left\{Y_{2}, W_{2}\right\}_{\text {num }}$}

From (5.25) and (5.20),

$$
W_{2, \sigma}^{\top}-W_{1, \sigma+\left[r_{b}-1\right]+p}^{\top} \geqslant 1-s_{b}-p^{\prime}
$$

which is a weak version of the first Burge condition (4.15). 


\subsection{7 $\left\{Y_{1}, W_{2}\right\}_{\text {num }}^{\prime}$ and $\left\{Y_{1}, W_{1}\right\}_{\text {num }}$}

From (5.23) and (5.16),

$$
W_{1, \sigma}^{\top}-W_{2, \sigma+\left[p-r_{b}-1\right]+p}^{\top} \geqslant 1-\left[p^{\prime}-s_{b}\right]-p^{\prime}
$$

which is a weak version of the second Burge condition (4.19).

\subsection{8 $\left\{Y_{2}, W_{2}\right\}_{\text {num }}^{\prime}$ and $\left\{Y_{2}, W_{1}\right\}_{\text {num }}$}

From (5.21) and (5.24),

$$
W_{1, \sigma}^{\top}-W_{2, \sigma+\left[p-r_{b}-1\right]}^{\top} \geqslant 1-\left[p^{\prime}-s_{b}\right]
$$

which is the second Burge condition (4.19).

The stronger condition in each of the above cases is one of the Burge conditions. Thus, when the denominator $z_{\text {den }}$ of the building block partition function $Z_{b b}$ is non-zero, then the numerator $z_{\text {num }}$ is also non-zero. The reverse is not true.

Note that the above result is similar but different from that in [7], where Zamolodchikov argues that 1 . The conformal block $\mathcal{B}_{n}^{\text {gen }}$ is a meromorphic function in $\Delta_{a}$, the conformal dimension of the Virasoro irrep that flows in a channel, and that $\mathcal{B}_{n}^{\text {gen }}$ has only simple poles at $\Delta_{a}=\Delta_{a_{r, s}}$, where $a_{r, s}=-\frac{1}{2}\left[r \alpha_{+}+s \alpha_{-}\right]$, and $\mathbf{2}$. If the fusion rules are satisfied, then the residue at each pole vanishes.

Our result is that 1 . When a summand in $Z_{b b}$ has a zero in the denominator, and the fusion rules are satisfied, then it also has a zero in the numerator. This is independent of Zamolodchikov's statement, since in the latter, the whole sum vanishes rather than just the summand with the zero in the denominator. 2. Zamolodchikov has argued that $\mathcal{B}_{n}^{\text {gen }}$ has only simple poles, while, as far as we can tell, summands in $Z_{b b}$ can have poles of order greater then 1.

\section{$6 \quad$ An Ising conformal block}

In this section, we set $p=3$ and $p^{\prime}=4$, so that the minimal model component $\mathcal{M}^{p, p^{\prime}}$ of the conformal field theory $\mathcal{M}^{p, p^{\prime}, \mathcal{H}}$ under consideration, is the Ising model. In this case, there are three primary fields to form conformal blocks from. They can be labeled as follows. $\{r, s\}=\{1,1\}$ is the identity operator $\mathbb{1},\{r, s\}=\{1,2\}$ is the spin operator $\sigma$ and $\{r, s\}=\{1,3\}$ is the thermal operator $\psi$. Explicit expressions for conformal blocks can be found in [15] and references therein. Consider the 6-point conformal block of $\sigma$ fields in figure 6 .

In this case, $\alpha_{+}=\sqrt{4 / 3}, \alpha_{-}=-\sqrt{3 / 4}$, and $\alpha_{1,2}=-\frac{1}{2} \alpha_{-}=-\frac{1}{2} \sqrt{3 / 4}$, and following [15],

$$
\begin{aligned}
& \left\langle\sigma\left(z_{0}\right) \sigma\left(z_{1}\right) \cdots \sigma\left(z_{5}\right)\right\rangle= \\
& \frac{1}{2} \prod_{i=1}^{3}\left(z_{2 i-2}-z_{2 i-1}\right)^{-\frac{1}{8}}\left(\sum_{\substack{t_{1}=1,1 \\
t_{2}, t_{3}=-1,1}} \prod_{i=1}^{3} t_{i} \prod_{1 \leqslant i<j \leqslant 3}\left(1-x_{i, j}\right)^{\frac{t_{i} t_{j}}{4}}\right)^{\frac{1}{2}},
\end{aligned}
$$




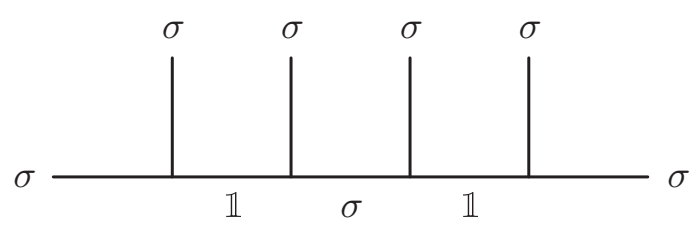

Figure 5. The comb diagram representation of the Ising 6-point conformal block discussed in section 6. All external lines correspond to vertex operator insertion of the spin operator $\sigma$. The internal channels carry the Virasoro irreps that correspond to the identity operator, the spin operator, then the identity operator.

where

$$
x_{i, j}=\frac{\left(z_{2 i-2}-z_{2 i-1}\right)\left(z_{2 j-2}-z_{2 j-1}\right)}{\left(z_{2 i-2}-z_{2 j-1}\right)\left(z_{2 j-2}-z_{2 i-1}\right)}
$$

setting the coordinates $z_{0}=0, z_{1}=q_{1} q_{2} q_{3}, z_{2}=q_{2} q_{3}, z_{3}=q_{3}, z_{4}=1, z_{5}=\infty$,

$$
x_{1,2}=\frac{-q_{1}\left(1-q_{2}\right)}{1-q_{1}}, \quad x_{1,3}=q_{1} q_{2} q_{3}, \quad x_{2,3}=\frac{q_{3}\left(1-q_{2}\right)}{1-q_{2} q_{3}} .
$$

The instanton partition function should equal the product of the Ising conformal block and a contribution from the Heisenberg algebra $\mathcal{H}^{16}$ Using e.g. [5], equation (1.9),

$$
Z=\left(\prod_{1 \leqslant i \leqslant j \leqslant 3}\left(1-q_{i} \cdots q_{j}\right)^{\frac{1}{8}}\right)\left\langle\sigma\left(z_{0}\right) \sigma\left(z_{1}\right) \cdots \sigma\left(z_{5}\right)\right\rangle
$$

Therefore

$$
\begin{aligned}
Z= & 1-\frac{1}{8} q_{1}-\frac{1}{8} q_{3}-\frac{5}{128} q_{1}^{2}-\frac{1}{8} q_{1} q_{2}+\frac{1}{64} q_{1} q_{3} \\
& -\frac{5}{128} q_{2}^{2}-\frac{1}{8} q_{2} q_{3}-\frac{5}{128} q_{3}^{2}+\cdots-\frac{453}{8192} q_{1}^{2} q_{2}^{2} q_{3}^{2}+\cdots
\end{aligned}
$$

Calculating the expansion of $Z$ up to degree 2 in each variable, we find that result coincides with the sum of non-zero terms in the instanton partition function. Using the notation

$$
\begin{aligned}
& Z_{\mathrm{Nek}}\left(Y_{1}, Y_{2}\left|Y_{3}, Y_{4}\right| Y_{5}, Y_{6}\right)= \\
& Z_{b b}\left(\varnothing, \varnothing \mid Y_{1}, Y_{2}\right) \cdot Z_{b b}\left(Y_{1}, Y_{2} \mid Y_{3}, Y_{4}\right) \cdot Z_{b b}\left(Y_{3}, Y_{4} \mid \varnothing, \varnothing\right)
\end{aligned}
$$

the $q_{1}^{2} q_{2}^{2} q_{3}^{2}$-term, as an example, is

$$
\begin{aligned}
Z_{\mathrm{Nek}}(2, \varnothing|\varnothing, 2| 2, \varnothing)+Z_{\mathrm{Nek}}(2, \varnothing|1,1| 2, \varnothing)+Z_{\mathrm{Nek}}(2, \varnothing|2, \varnothing| 2, \varnothing) & \\
& +Z_{\mathrm{Nek}}(2, \varnothing|2, \varnothing| 1+1, \varnothing)+Z_{\mathrm{Nek}}(2, \varnothing|1,1| 1+1, \varnothing)=-\frac{453}{8192}
\end{aligned}
$$

while all other terms, that satisfy Proposition 4.1 and the condition $\left|Y_{1}\right|+\left|Y_{2}\right|=\left|Y_{3}\right|+\left|Y_{4}\right|=$ $\left|Y_{5}\right|+\left|Y_{6}\right|=2$, vanish.

\footnotetext{
${ }^{16}$ The contribution of the Heisenberg algebra $\mathcal{H}$ is often referred to as the $\mathrm{U}(1)$ factor.
} 


\section{An explanation, based on a conjecture, of why we obtain $\mathcal{M}^{p, p^{\prime}, \mathcal{H}}$ con- formal blocks}

As mentioned in section 1, there is a proof of AGT in the context of conformal blocks in $\mathcal{M}^{\text {gen }, \mathcal{H}}$ with non-degenerate intermediate Virasoro representations in [5]. In this subsection, we use 1. results from [5], 2. Proposition 4.1 of section 4, 3. that the generating function of Burge pairs is the character of $\mathcal{H}_{r, s}^{p, p^{\prime}, \mathcal{H}}[10,11]$, and 4. Conjecture 7.1 below, to explain why restricting the summation to Burge pairs as in (1.4) leads to conformal blocks in $\mathcal{M}^{p, p^{\prime}, \mathcal{H}}$. Proving 3 would amount to proving that restricting to Burge pairs leads to conformal blocks in $\mathcal{M}^{p, p^{\prime}, \mathcal{H}}$, but this is beyond the scope of this work.

Consider the Verma module $\mathcal{H}_{a}$ over $\mathcal{V}^{\text {gen }} \oplus \mathcal{H}$ generated by highest-weight vector $|a\rangle$,

$$
L_{k}|a\rangle=a_{k}|a\rangle=0, \quad k>0, \quad L_{0}|a\rangle=\Delta_{a}|a\rangle,
$$

where $L_{k}$, and $a_{k}, k \in \mathbb{Z}$, are generators of $\mathcal{V}^{\text {gen }}$ and $\mathcal{H}$, respectively, and

$$
\Delta_{a}=\frac{1}{4}\left(b_{\text {gen }}+\frac{1}{b_{\text {gen }}}\right)^{2}-a^{2}, \quad b_{\text {gen }}=\left(\frac{\epsilon_{2}}{\epsilon_{1}}\right)^{\frac{1}{2}} .
$$

Conformal blocks are defined in terms of vertex operators $\mathcal{O}_{\mu}(z): \mathcal{H}_{a} \mapsto \mathcal{H}_{b}$, that in turn are defined by the commutation relations

$$
\left[L_{k}, \mathcal{O}_{\mu}(z)\right]=z^{k+1} \partial_{z} \mathcal{O}_{\mu}(z)+i(k+1) \Delta_{\mu^{\prime}} q^{k} \mathcal{O}_{\mu}(q), \quad \mu^{\prime}=\mu-\frac{\epsilon_{1}+\epsilon_{2}}{2}
$$

as well as

$$
\left[a_{k}, \mathcal{O}_{\mu}(z)\right]=i \mu z^{k} \mathcal{O}_{\mu}(z), \quad k<0, \quad\left[a_{k}, \mathcal{O}_{\mu}(z)\right]=i(Q-\mu) z^{k} \mathcal{O}_{\mu}(z), \quad k>0
$$

AGT was proven in [5] for generic central charge $c_{\text {gen }}$, in the following sense

Proposition 7.1 Following [5], there exists an orthogonal basis $J_{\vec{Y}} \in \mathcal{H}_{a}$ labeled by pairs of Young diagrams such that the matrix elements of vertex operator $\mathcal{O}_{\mu}$ satisfy

$$
\frac{\left\langle J_{\vec{Y}}\left|\mathcal{O}_{\mu}\right| J_{\vec{W}}\right\rangle}{\left\langle J_{\vec{\varnothing}}\left|\mathcal{O}_{\mu}\right| J_{\vec{\varnothing}}\right\rangle}=z_{\text {num }}(\vec{a}, \vec{Y}|\mu| \vec{b}, \vec{W})
$$

where $\vec{a}=\{a,-a\}, \vec{b}=\{b,-b\}$.

From this proposition, it follows that

$$
\left\langle J_{\vec{Y}} \mid J_{\vec{W}}\right\rangle=z_{\text {norm }}(\vec{a}, \vec{Y}) \delta_{\vec{Y}, \vec{W}}
$$

The vectors $J_{\vec{Y}}$ can be written in the standard basis of the Verma module,

$$
J_{\vec{Y}}=\sum_{\lambda, \mu} C_{\vec{Y}}^{\lambda, \mu} L_{-\lambda_{1}} L_{-\lambda_{2}} \cdots a_{-\mu_{1}} a_{-\mu_{2}} \cdots|a\rangle,
$$

where the summation is over partition pairs $\{\lambda, \mu\}$ such that $|\lambda|+|\mu|=\left|Y_{1}\right|+\left|Y_{2}\right|$. The coefficients $C_{\vec{Y}}^{\lambda, \mu}$ depend on the parameters $a, b_{\text {gen }}$. In [5, Corollary 3.8], it was proven that $C_{\vec{Y}}^{\lambda, \mu}$ is a polynomial in $a$. In this section, we need the following conjecture ${ }^{17}$

\footnotetext{
${ }^{17}$ This conjecture is not original to this work. It is standard in the community, although not written in the literature.
} 
Conjecture 7.1 The coefficients $C_{\vec{Y}}^{\lambda, \mu}$ are Laurent polynomials in $b_{\text {gen }}$.

Conjecture 7.1 is motivated by the explicit examples of the vectors $J_{\vec{Y}}[5]$. Further motivation is provided by the relation between Jack symmetric functions $J_{Y}^{\alpha}[16]$ and $J_{\vec{Y}}$, for $\alpha=b_{\text {gen }}^{2}$ [5]. Namely, from Macdonald's conjectures, proved by Haiman [17], the coefficients of $J_{Y}^{\alpha}$, in the standard basis, are polynomial in $\alpha$, so it is natural to expect that $J_{\vec{Y}}$ satisfies an analogous property.

Assuming Conjecture 7.1, we can set $b_{\text {gen }} \rightarrow b_{p, p^{\prime}}$ and $a \rightarrow a_{r, s}$, as defined in subsection 3.2. Thus, we can consider $J_{\vec{Y}}$ as vectors in the module over $\mathcal{H} \oplus \mathcal{V}^{p, p^{\prime}}$. In this case, the Verma module $\mathcal{H}_{a}$ becomes reducible, the maximal submodule $\operatorname{Ker}_{a}$ is a kernel of the Shapovalov form $\langle\cdot \mid \cdot\rangle$ on $\mathcal{H}_{a}$, and the irreducible quotient is $\mathcal{H}_{r, s}^{p, p^{\prime}, \mathcal{H}}=\mathcal{H}_{r, s}^{p, p^{\prime}} \otimes \mathcal{F}$.

It was follows from (4) and (7.6) that $\vec{Y}$ satisfy condition (4.1) if and only if the vector $J_{\vec{Y}}$ is not in the kernel $\operatorname{Ker}_{a}$. Then to each Burge pair $\vec{Y}_{\text {Burge }}$ one can consider $J_{\vec{Y}}$ as an element of quotient $\mathcal{H}_{r, s}^{p, p^{\prime}, \mathcal{H}}$. The vectors $J_{\vec{Y}} \in \mathcal{H}_{r, s}^{p, p^{\prime}, \mathcal{H}}$, for a Burge pair $\vec{Y}_{\text {Burge }}$, are linearly independent since they are orthogonal. It was proven in $[10,11]$, that the generating function of Burge pairs give the character of $\mathcal{H}_{r, s}^{p, p^{\prime}, \mathcal{H}}$. Therefore the vectors $J_{\vec{Y}_{\text {Burge }}}$ form a basis in $\mathcal{H}_{r, s}^{p, p^{\prime}, \mathcal{H}}$. This is the point of this subsection.

Finally we note that the norms and matrix elements of $\mathcal{O}_{\mu}$ depend on the parameters $\left\{a, b, \mu, \epsilon_{1}, \epsilon_{2}\right\}$ algebraically. Therefore, since (7.5) was proven for a generic central charge, it holds for the $\mathcal{M}^{p, p^{\prime}, \mathcal{H}}$ models, and the expression that we obtain for $\mathcal{B}_{n}^{p, p^{\prime}, \mathcal{H}}$, by summing over Burge pairs, holds.

\section{Generic model conformal blocks with Degenerate intermediate repre- sentations}

In generic models with a generic central charge $c_{\text {gen }}, z_{\text {norm }}[\vec{a}, \vec{Y}]$ can have zeros due to degenerate $\mathcal{V}^{\text {gen }}$ representations in the intermediate channels. Since $\vec{a}=\{a,-a\}$, setting $2 a=2 a_{r, s}=-r \beta_{+}-s \beta_{-}$, we can study these zeros just as in section 4 . Since the central charge is generic we have

$$
C_{+} \beta_{+}+C_{-} \beta_{-}=0
$$

if and only if $C_{+}=C_{-}=0$, and only the $\left\{Y_{1}, Y_{2}\right\}_{\text {den }}$ factors can be zero. Proceeding from the two zero-conditions (8.1), we obtain

Proposition $8.1 z_{\text {norm }}[\vec{a}, \vec{Y}] \neq 0$ if and only if $Y_{2, \sigma}^{\top}-Y_{1, \sigma+r-1}^{\top} \geqslant 1-s$

The proof of Proposition 8.1 follows the same line of arguments as the proof of Proposition 4.1 in section 4. From Proposition 8.1, we obtain

\section{Proposition 8.2}

$$
\mathcal{B}_{n}^{\operatorname{degen}, \mathcal{H}}=\sum_{\vec{Y}^{1}, \cdots, \vec{Y}^{n}}^{\prime} \prod_{\iota=1}^{n+1} q_{\iota}^{\left|\vec{Y}^{\iota}\right|} Z_{b b}\left(\vec{a}^{\iota-1}, \vec{Y}^{\iota-1}\left|\mu^{\iota}\right| \vec{a}^{\iota}, \vec{Y}^{\iota},\right)
$$

where $\mathcal{B}_{n}^{\text {degen, } \mathcal{H}}$ is an n-channel generic model conformal block, such that some of the channels carry degenerate intermediate representations, and $\sum^{\prime}$ indicates that, for channels 
that carry degenerate representations, the sum is restricted to partition pairs that satisfy Proposition 8.1.

The proof of Proposition 8.2 is based on the same line of arguments as in section 7 but without requiring a conjecture analogous to Conjecture 7.1. Indeed, since the coefficients of $J_{\vec{Y}}$ are polynomial in $a$, we can set $a=a_{r, s}$ in (7.7). The vectors $J_{\vec{Y}}$ for which $z_{\text {norm }}[\vec{a}, \vec{Y}]=$ 0 , belong to the kernel of the Shapovalov form on the Verma module $\mathcal{H}_{a}$. Let $\mathcal{H}_{r, s}^{\text {gen }}$ denote the irreducible quotient of $\mathcal{H}_{a}$. The vectors $J_{\vec{Y}}$, where $\vec{Y}$ satisfy Proposition 8.1, project to the module $\mathcal{H}_{r, s}^{\text {gen }}$. In [11], Feigin et al. proved that the generating function of the $\vec{Y}$ pairs that satisfy Proposition 8.1 is the character of $\mathcal{H}_{r, s}^{\text {gen }}$, therefore the corresponding vectors $J_{\vec{Y}}$ form a basis in $\mathcal{H}_{r, s}^{\text {gen }}$. Using (7.5) and (7.6), we obtain the expression (8.2) for the conformal block for degenerate representations.

\section{Comments and remarks}

\section{$9.1 \quad q-\mathfrak{g l}_{\infty}$ Ding-Iohara}

Let $\mathcal{E}$ be the algebra called $q$-deformed $\mathfrak{g l}_{\infty}$ in [11], and Ding-Iohara in [18]. ${ }^{18}$ Following [18], operators in the rank-2 representations $\mathcal{F}_{u_{1}} \otimes \mathcal{F}_{u_{2}}$ of $\mathcal{E}$ generate the sum of a $q$-deformed Virasoro algebra and a $q$-deformed Heisenberg algebra. On the other hand, following [11] [Theorem 3.8], for special values of parameters $u_{1}$ and $u_{2}$, as well as $q_{1}$ and $q_{3}$ of $\mathcal{E}$, this representation has a sub-representation with a basis labeled by Burge pairs. In is natural to expect that in the limit $q_{1}, q_{3} \rightarrow \infty$, the basis constructed in [11] reduces to the basis $J_{\vec{Y}}$ described in section 7 .

\subsection{Higher-rank AGT-W}

AGT was extended to theories based on the higher rank algebras $\mathcal{W}_{N} \oplus \mathcal{H}, N>2$, by Wyllard in [19], and by Mironov and Morozov in [20]. In this note, we chose to simplify the presentation by focusing on Virasoro minimal models, but we expect that our analysis extends without essential modification to minimal models based on $\mathcal{W}_{N}$ algebras with $N>2$. We conjecture that the restricted partitions that are relevant to these extended cases are those that appeared in [11].

\subsection{The work of Alkalaev and Belavin}

In [21], Alkalaev and Belavin independently suggested the Virasoro result in (1.4) in the 4-point conformal block case. They proved a proposition equivalent to 4.1, made the same comment on conformal blocks in generic models with degenerate intermediate representations as in section 8, albeit without proving an analogue of Proposition 8.2 and made the same $W_{N}$ conjecture as in subsection 9.2.

\footnotetext{
${ }^{18}$ Also called 'quantum toroidal $\mathfrak{g l}(1)$ algebra', 'elliptic Hall algebra', etc. in the literature.
} 


\subsection{Previous works on AGT in minimal models}

There are two previous works on AGT in minimal models that we are aware of. In [8], Santachiara and Tanzini identify Moore-Read wave functions, which are minimal model conformal blocks of $\{r, s\}=\{1,2\}$ and $\{2,1\}$ vertex operators, with Nekrasov instanton partition functions, AGT is applied without modification to these conformal blocks and ill-defined expressions are made well-defined using a deformation scheme, as outlined in subsection 1.3. In [22], Estienne, Pasquier, Santachiara and Serban interpret $W_{n} \oplus \mathcal{H}$ minimal model conformal blocks of $\{r, s\}=\{1,2\}$ and $\{2,1\}$ vertex operators as wave functions of a trigonometric Calogero-Sutherland models with non-trivial braiding properties, and find that the excited states are characterized by $(n+1)$-partitions, just as in AGT. While Estienne et al. use different notation from ours, preliminary checks indicate that their partitions can be translated to the Burge pairs used in this note, for $n=2$, and $\{r, s\}=\{1,2\}$ or $\{2,1\}$.

\subsection{Geometry}

Let $\mathcal{M}(r, N)$ be the moduli space of $\mathrm{U}(r)$ instantons on $\mathbb{R}^{4}$. The instanton partition function for $\prod_{i=1}^{n} \mathrm{U}(2)$, gauge theory equals the generating function of equvariant integrals over $\mathcal{M}\left(2, N_{1}\right) \times \cdots \times \mathcal{M}\left(2, N_{n}\right)$, where the equivariant integral is taken with respect to the torus $\mathbb{T}=\left(\mathbb{C}^{*}\right)^{2} \times\left(\mathbb{C}^{*}\right)_{1}^{2} \times\left(\mathbb{C}^{*}\right)_{2}^{2} \times \cdots \times\left(\mathbb{C}^{*}\right)_{n}^{2}$, where the first $\left(\mathbb{C}^{*}\right)^{2}$ acts on $\mathbb{C}^{2}$, and $\left(\mathbb{C}^{*}\right)_{i}^{2}$ acts on the $i$-th instanton moduli space $\mathcal{M}\left(2, N_{i}\right)$ by constant gauge transformation. These equvariant integrals are computed using localization and is given by the torus fixed points. These points were labeled by $n$ pairs of Young diagrams $\vec{Y}^{1}, \cdots, \vec{Y}^{n}$. The parameters $\epsilon_{1}$, $\epsilon_{2}$, and $\vec{a}^{i}$ are the coordinates on $\mathrm{t}=\operatorname{Lie}(\mathbb{T})$. In the $\mathcal{M}^{p, p^{\prime}, \mathcal{H}}$ case, $\epsilon_{1}$ and $\epsilon_{2}$ are linearlydependent, and $a_{j}^{i}$ is given by (3.7). Geometrically, this means that we are considering the one-dimensional subgroup $\mathbb{C}_{\epsilon_{1}, \epsilon_{2}, \vec{a}^{i}}^{*} \subset \mathbb{T}$.

The function $z_{\text {norm }}[\vec{a}, \vec{Y}]$ is the determinant of the vector field with coordinates $\left\{\epsilon_{1}, \epsilon_{2}\right.$, $\left.a_{1}, a_{2}\right\}$ on the tangent space of the point labeled by $\vec{Y}$. The condition $z_{\text {norm }}[\vec{a}, \vec{Y}] \neq 0$ is equivalent to the fact that corresponding point is an isolated fixed point of the one dimensional torus $\mathbb{C}_{\epsilon_{1}, \epsilon_{2}, \vec{a}^{i}}^{*}$. Therefore, summing over Burge pairs is equivalent to summing over the isolated torus fixed points.

\section{Acknowledgments}

We wish to thank the Simons Center for Geometry and Physics for hospitality where this work was started, Dr S Corteel and Laboratoire d'Informatique Algorithmique: Fondements et Applications, Paris 7, for hosting OF while it was completed. MB was supported in part by RFBR grants 12-02-01092-a, 13-01-90614, and OF was supported by Australian Research Council and the Fondation Sciences Mathématiques de Paris. We wish to thank V Fateev, B Feigin, I Kostov, R Santachiara, D Serban and A Tanzini for discussions and useful remarks. 
Open Access. This article is distributed under the terms of the Creative Commons Attribution License (CC-BY 4.0), which permits any use, distribution and reproduction in any medium, provided the original author(s) and source are credited.

\section{References}

[1] L.F. Alday, D. Gaiotto and Y. Tachikawa, Liouville Correlation Functions from Four-dimensional Gauge Theories, Lett. Math. Phys. 91 (2010) 167 [arXiv:0906.3219] [INSPIRE].

[2] A.A. Belavin, A.M. Polyakov and A.B. Zamolodchikov, Infinite Conformal Symmetry in Two-Dimensional Quantum Field Theory, Nucl. Phys. B 241 (1984) 333 [inSPIRE].

[3] N.A. Nekrasov, Seiberg-Witten prepotential from instanton counting, Adv. Theor. Math. Phys. 7 (2004) 831 [hep-th/0206161] [INSPIRE].

[4] A. Mironov, A. Morozov and S. Shakirov, A direct proof of AGT conjecture at $\beta=1$, JHEP 02 (2011) 067 [arXiv: 1012.3137] [INSPIRE].

[5] V.A. Alba, V.A. Fateev, A.V. Litvinov and G.M. Tarnopolskiy, On combinatorial expansion of the conformal blocks arising from AGT conjecture, Lett. Math. Phys. 98 (2011) 33 [arXiv: 1012.1312] [INSPIRE].

[6] R. Santachiara, private communication.

[7] A.B. Zamolodchikov, Conformal symmetry in two-dimensions: an explicit recurrence formula for the conformal partial wave amplitude, Commun. Math. Phys. 96 (1984) 419 [INSPIRE].

[8] R. Santachiara and A. Tanzini, Moore-Read Fractional Quantum Hall wavefunctions and SU(2) quiver gauge theories, Phys. Rev. D 82 (2010) 126006 [arXiv:1002.5017] [INSPIRE].

[9] W.H. Burge, Restricted partition pairs, J. Comb. Theory. Ser. A 63 (1993) 210.

[10] O. Foda, K.S.M. Lee and T.A. Welsh, A Burge tree of Virasoro type polynomial identities, Int. J. Mod. Phys. A 13 (1998) 4967 [q-alg/9710025] [INSPIRE].

[11] B. Feigin, E. Feigin, M. Jimbo, T. Miwa and E. Mukhin, Quantum continuous $\mathfrak{g l}_{\infty}$ : Tensor products of Fock modules and $\mathcal{W}_{n}$-characters, Kyoto J. Math 51 (2011) 365. [arXiv:1002.3113].

[12] S. Kanno, Y. Matsuo and H. Zhang, Extended Conformal Symmetry and Recursion Formulae for Nekrasov Partition Function, JHEP 08 (2013) 028 [arXiv:1306.1523] [INSPIRE].

[13] B. Nienhuis, Coulomb gas representations of phase transitions in two dimensions, Phase Trans. Critical Phenom. 11 (1987) 1.

[14] Vl.S. Dotsenko and V.A. Fateev, Conformal algebra and multipoint correlation functions in $2 D$ statistical models, Nucl. Phys. B 240 (1984) 312.

[15] E. Ardonne and G. Sierra, Chiral correlators of the Ising conformal field theory, J. Phys. A 43 (2010) 505402 [arXiv:1008.2863] [inSPIRE].

[16] I.G. Macdonald, Symmetric functions and Hall polynomials, 2nd edition, Clarendon Press Oxford (1995).

[17] M. Haiman, Hilbert schemes, polygraphs and the Macdonald positivity conjecture, J. Am. Math Soc. 14 (2001) 941 [math/0010246]. 
[18] B. Feigin, A. Hoshino, J. Shibahara, J. Shiraishi and S. Yanagida, Kernel function and quantum algebras, RIMS kôkyûroku 1689 (2010) 133 [arXiv:1002.2485].

[19] N. Wyllard, A(N-1) conformal Toda field theory correlation functions from conformal $N=2$ $\mathrm{SU}(N)$ quiver gauge theories, JHEP 11 (2009) 002 [arXiv:0907.2189] [INSPIRE].

[20] A. Mironov and A. Morozov, On AGT relation in the case of U(3), Nucl. Phys. B 825 (2010) 1 [arXiv:0908.2569] [InSPIRE].

[21] K.B. Alkalaev and V.A. Belavin, Conformal blocks of $W_{N}$ Minimal Models and AGT correspondence, arXiv:1404.7094 [INSPIRE].

[22] B. Estienne, V. Pasquier, R. Santachiara and D. Serban, Conformal blocks in Virasoro and W theories: Duality and the Calogero-Sutherland model, Nucl. Phys. B 860 (2012) 377 [arXiv:1110.1101] [INSPIRE]. 